\title{
A diffusiophoretic mechanism for ATP-driven transport without motor proteins
}

\author{
Beatrice Ramm ${ }^{1,4,6}$, Andriy Goychuk ${ }^{2,6}$, Alena Khmelinskaia ${ }^{1,5}$, Philipp Blumhardt \\ Hiromune Eto ${ }^{1}{ }^{1}$, Kristina A. Ganzinger ${ }^{3}{ }^{3}$, Erwin Frey ${ }^{2 \bowtie}$ and Petra Schwille ${ }^{1}{ }^{\bowtie}$
}

The healthy growth and maintenance of a biological system depends on the precise spatial organization of molecules within the cell through the dissipation of energy. Reaction-diffusion mechanisms can facilitate this organization, as can directional cargo transport orchestrated by motor proteins, by relying on specific protein interactions. However, transport of material through the cell can also be achieved by active processes based on non-specific, purely physical mechanisms, a phenomenon that remains poorly explored. Here, using a combined experimental and theoretical approach, we discover and describe a hidden function of the Escherichia coli MinDE protein system: in addition to forming dynamic patterns, this system accomplishes the directional active transport of functionally unrelated cargo on membranes. Remarkably, this mechanism enables the sorting of diffusive objects according to their effective size, as evidenced using modular DNA origami-streptavidin nanostructures. We show that the diffusive fluxes of MinDE and non-specific cargo couple via density-dependent friction. This non-specific process constitutes a diffusiophoretic mechanism, as yet unknown in a cell biology setting. This nonlinear coupling between diffusive fluxes could represent a generic physical mechanism for establishing intracellular organization.

patiotemporal organization of cells generally emerges through redistribution and transport of molecules via motor proteins ${ }^{1}$, self-assembling cytoskeletal elements ${ }^{2}$ or self-organizing reaction-diffusion systems ${ }^{3}$. Coupling of cargo to energy-dissipating NTPases that drive the transport is usually mediated by specific protein-protein interactions. Non-specific coupling of biomolecules, by contrast, is poorly explored in biology and, so far, only a few examples of molecular transport based on purely physical mechanisms have been reported. For example, a study in mouse oocytes showed that actin-coated vesicles generated a pressure gradient that positioned large objects like the nucleoid in the cell centre ${ }^{4,5}$. In the Caenorhabditis elegans zygote, cortical flows were shown to couple to the PAR reaction-diffusion system via advective transport ${ }^{6}$. Another recent example comes from in vitro studies of the Escherichia coli Min system ${ }^{7,8}$.

The Min system, a paradigmatic model for pattern formation in biology, regulates the site of cell division in E. coli ${ }^{9-12}$. The core of this reaction-diffusion system consists of only two proteins, the ATPase MinD and the ATPase activating protein MinE, which interact and reversibly bind to the membrane ${ }^{11,13}$. Despite this simple reaction scheme, the Min system exhibits rich dynamics that have been explored in vivo ${ }^{9-11,14}$, reconstituted in vitro ${ }^{13,15,16}$ and described by physical theories ${ }^{17-21}$. In the rod-shaped E. coli, MinDE oscillate from pole to pole ${ }^{10,11,14}$. In vitro, MinDE proteins form travelling surface waves or quasi-stationary patterns on planar artificial membranes ${ }^{13,15,16}$ and exhibit oscillations when geometrically confined ${ }^{22,23}$. These dynamics can provide spatial cues for particular proteins. MinC specifically binds to MinD and thus follows its movements ${ }^{10,22,24-26}$. In turn, MinC constrains the localization of the main divisome protein FtsZ by inhibiting its polymerization ${ }^{27,28}$.

Besides this well-described patterning by specific interactions with clear physiological evidence, MinDE self-organization has recently shown an intriguing hidden function in vitro: MinDE regulated the localization of unrelated membrane-bound molecules in space and time in the absence of MinC/FtsZ ${ }^{7,8}$. These results suggested that MinDE oscillations could further enhance cell division by prepositioning membrane proteins to the cell middle ${ }^{7}$. However, the underlying physics and the broader biological implications have remained unknown.

Here, through a joint experimental and theoretical investigation, we have deciphered the physical mechanism underlying this non-specific transport phenomenon. We quantitatively probed MinDE-dependent transport using a synthetic membrane-bound cargo based on composite DNA nanostructures. We discerned how the effective size (that is, membrane footprint) and diffusion coefficient of the cargo, as well as the type of MinDE patterns, determine the transport that takes place. Exploiting these effects, we revealed that MinDE can even spatially sort different cargo species. Theoretical analysis of these data demonstrated a diffusiophoretic effect: an effective density-dependent inter-particle friction creates cargo transport along the diffusive fluxes of MinD proteins. This type of NTPase-driven diffusiophoresis might represent a generic active transport mechanism in cells that neither requires motor proteins nor specific protein interactions. As such, it might be particularly important for prokaryotes and could have been prevalent in early stages of life on Earth.

\section{Probing MinDE-induced transport with a synthetic cargo}

We set out to understand how MinDE dynamics can spatiotemporally regulate other membrane-bound, yet unrelated molecules (henceforth referred to as non-specific 'cargo') into patterns and gradients $^{7,8}$. Specifically, we asked whether cargo transport can arise from MinDE patterns per se via thermodynamic forces, or whether it requires active processes and non-equilibrium particle fluxes.

'Max Planck Institute of Biochemistry, Martinsried, Germany. ${ }^{2}$ Arnold-Sommerfeld-Center for Theoretical Physics and Center for NanoScience, Ludwig-Maximilians-Universität München, Munich, Germany. ${ }^{3}$ Living Matter Program, AMOLF, Amsterdam, Netherlands. ${ }^{4}$ Present address: Department of Physics, Princeton University, Princeton, NJ, USA. ${ }^{5}$ Present address: University of Washington, Institute for Protein Design, Seattle, WA, USA. ${ }^{6}$ These authors contributed equally: Beatrice Ramm, Andriy Goychuk.凶e-mail: frey@lmu.de; schwille@biochem.mpg.de 
To experimentally address this question and test possible mechanisms, we set up a highly controllable minimal in vitro platform. To this end, we reconstituted MinDE pattern formation on supported lipid bilayers (SLBs) ${ }^{13}$. For simplicity, we first chose conditions under which initially chaotic, laterally moving MinDE waves transition into quasi-stationary labyrinth patterns ${ }^{16}$. To quantitatively assess the interaction between MinDE and cargo molecules on the membrane, we employed a synthetic cargo: a composite object consisting of a DNA origami nanostructure as scaffold and streptavidin molecules that serve as modular building blocks and connectors to the membrane (Fig. 1a). In particular, the origami $^{29}$ featured 7 dyes on the upper facet for visualization and 42 sites at the bottom facet that could be specifically addressed for the incorporation of biotinylated oligonucleotide handles. These handles in turn bound to streptavidin coupled to biotinylated lipids in the SLB.

When we initiated MinDE self-organization with ATP in the presence of this synthetic cargo, the cargo components-that is, the origami structures and streptavidin-reorganized into patterns from an initially homogeneous state (Fig. 1b,c, Supplementary Fig. 1 and Supplementary Video 1). As expected, the origami and streptavidin co-localized. Accordingly, in the following, origami fluorescence serves as a proxy for cargo localization. For the entire duration of the experiment, cargo always gathered in MinD-depleted regions, forming patterns that were anti-correlated to the MinDE patterns (Fig. 1b,c). Similarly, when we altered the established MinDE/cargo patterns by adding more MinE, the cargo channel reflected the changes in MinDE patterns, moving in an anti-correlated fashion (Supplementary Fig. 2 and Supplementary Video 2). By contrast, when MinE (which stimulates MinD ATP hydrolysis) was omitted, MinD and the cargo molecules remained uniformly distributed (Extended Data Fig. 1). These findings indicate that the spatial heterogeneity of the cargo is not caused by depletion attraction, such as in filament bundling ${ }^{30}$. For one, depletion attraction should lead to aggregation of large particles (cargo), even in a homogeneous field of smaller particles $(\mathrm{MinD})^{31}$. Furthermore, depletion attraction would imply a preferred agglomeration of cargo in regions of high MinD density, which we also did not observe (Supplementary Information). Hence, our data demonstrate that cargo transport is active, because it requires the presence of MinDE and ATP, and thus active MinDE self-organization.

\section{Effective cargo size determines the extent of cargo demixing}

Having shown that MinDE redistribute our synthetic cargo, we next exploited the modular nature of our cargo to systematically vary its interaction with MinDE. MinD and lipid-anchored streptavidin both form a monomolecular layer of height $\sim 5 \mathrm{~nm}$ on membranes $^{32,33}$, whereas the lower facet of the origami scaffold lies at $\sim 5-11 \mathrm{~nm}$ above the membrane (Supplementary Note 1). MinDE thus move on the membrane below the altitude of the origami scaffold and should mainly interact with the membrane-bound streptavidin. Hence, varying the number of streptavidin $(n \in\{1,2,5$, $15,28,42\}$ ) bound to the origami scaffold enables fine control over a large dynamic range of the membrane footprint or effective size of the cargo (henceforth cargo- $n$, Fig. 1 ). In this way, we modulated the interaction of the cargo- $n$ with MinDE, as well as its diffusion on the membrane.

To quantitatively assess the interaction between MinDE and the respective cargo, we analysed the resulting, final quasi-stationary patterns (Fig. 1d and Extended Data Fig. 2). As a measure for molecule enrichment, we determined the Michelson contrast, $\left(I_{\max }-I_{\min }\right) /\left(I_{\max }+I_{\min }\right)$, of the fluorescence images on a scale from zero for a homogeneous distribution to one for a binary distribution. Cargo patterns became much sharper with increasing cargo size (Fig. 1e and Extended Data Fig. 2). This increase in the contrast of cargo patterns was accompanied by sharper and also narrower MinDE patterns, as indicated by an increased region of pixels classified as MinD minima (Fig. 1f,g). Thus, MinDE dynamics dictate the localization of cargo on the membrane in a size-dependent manner, and are in turn also impacted by their presence. At first glance, these results could be interpreted as simply being a consequence of steric repulsion between cargo and MinDE. However, although a static gradient of accessible space would indeed induce a gradient of cargo molecules, this effect should be equal for all cargo species, irrespective of their effective size (Supplementary Information). Therefore, even though the diffusion of MinD on the membrane under the given conditions is very slow $\left(D=0.013 \mu \mathrm{m}^{2} \mathrm{~s}^{-1}\right)^{25}$, we conclude that MinD proteins do not simply act as immobile obstacles on the membrane that would bias cargo diffusion via static volume exclusion, a second option for a thermodynamic force.

\section{Thermodynamic forces cannot explain cargo transport by MinDE}

As our experimental data disqualified both depletion attraction and static volume exclusion as possible explanations for cargo redistribution, we wondered whether mobile MinD proteins could effectively repel cargo in a size-dependent manner by imposing local constraints on the entropy of mixing. To test this third (thermodynamic) hypothesis, we formulated a fully quantitative FloryHuggins theory $(\mathrm{FH})$ without fitting parameters. Specifically, each origami scaffold crosslinks $n$ streptavidin into a passive polymer-like cargo (Fig. 2a), while the remaining free streptavidin (for $n<15$; Extended Data Fig. 2) behave independently (Supplementary Information). Given these constraints, we characterized the membrane in terms of local surface densities of $c_{\mathrm{g}}$ cargo, $c_{\mathrm{s}}$ free streptavidin and $c_{\mathrm{p}}$ MinD (Supplementary Table 1), assuming a well-mixed lattice gas on the microscopic scale. On the mesoscopic scale, we then asked 'what is the equilibrium distribution of passive particles (cargo and free streptavidin) in the presence of a heterogeneous distribution of active MinD proteins?' To answer this question, we used our FH model to calculate the corresponding chemical

Fig. 1 | MinDE-driven cargo demixing depends on the effective size (membrane footprint) of the cargo. a, Schematic of the synthetic

membrane-anchored cargo consisting of a DNA origami scaffold ( 20 -helix bundle; $110 \times 16 \times 8 \mathrm{~nm}$ ) and streptavidin building blocks. The DNA origami nanostructure illustrates the position of 7 dyes at the upper facet and 42 addressable sites for incorporation of biotinylated oligonucleotides at the lower facet. Biotinylated oligonucleotides bind to lipid-anchored streptavidin on the SLB. MinDE self-organize by concerted attachment and detachment to and from the membrane powered by ATP hydrolysis into ADP and $P_{i}$ (inorganic phosphate). The self-organization assay is performed in an open chamber. b,c, Representative time series (b) and kymograph (along the line selection in $\mathbf{b}$ ) (c) of MinDE self-organization inducing patterns of cargo-2 ( $1 \mu \mathrm{M}$ MinD (30\% EGFP-MinD), $1.5 \mu$ M MinE-His, $0.1 \mathrm{nM}$ origami-Cy5 with two biotinylated oligonucleotides, Alexa568-streptavidin). d, Representative images and fluorescence intensity line plots (smoothed) of established MinDE labyrinth patterns and anti-correlated DNA origami and streptavidin patterns when no origami, cargo-2, cargo-15 or cargo-42 is present. The contrast of the resulting patterns and size of the MinD minima increase with increasing number of incorporated streptavidin per cargo. e-g, Box plots of the contrast of cargo (e), MinD patterns (f) and the fraction of pixels classified as MinD minima ( $\mathbf{g}$ ) when no origami, cargo-1, cargo-2, ..., or cargo-42 is present. Lines in box plots are medians, box limits are quartiles 1 and 3 , whiskers are $1.5 \times$ interquartile range (IQR) and points are outliers. Data are from at least two independent experiments with total numbers of analysed images per condition $N(\mathrm{No}$ origami $)=32, N($ Cargo- 1$)=96, N($ Cargo -2$)=41, N($ Cargo -5$)=32, N($ Cargo-15) $=94, N($ Cargo -28$)=32, N($ Cargo -42$)=87$. Scale bars, $50 \mu m(\mathbf{b}, \mathbf{c}, \mathbf{d})$. 
potentials $\mu_{i}$ for each species (Supplementary Information). Furthermore, we assumed that the passive particles adopt a thermal equilibrium state with vanishing chemical potential gradients $\left(\nabla \mu_{\mathrm{g}}=\nabla \mu_{\mathrm{s}}=0\right)$ in an adiabatic response to the imposed steady-state distribution of active particles $\left(\nabla \mu_{\mathrm{p}} \neq 0\right)$. Our theoretical analysis shows that entropic mixing effects can, in principle, create a

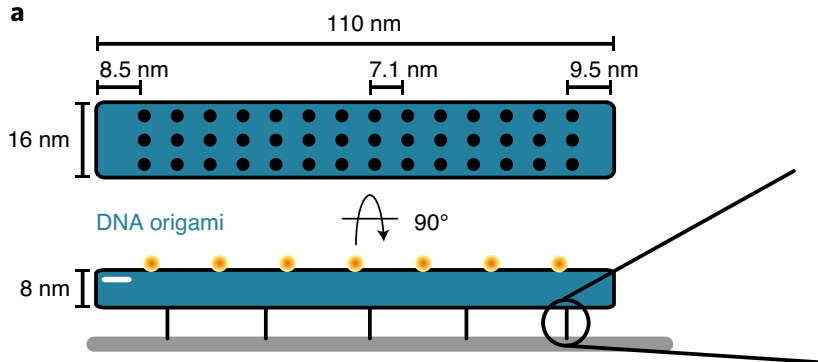

b

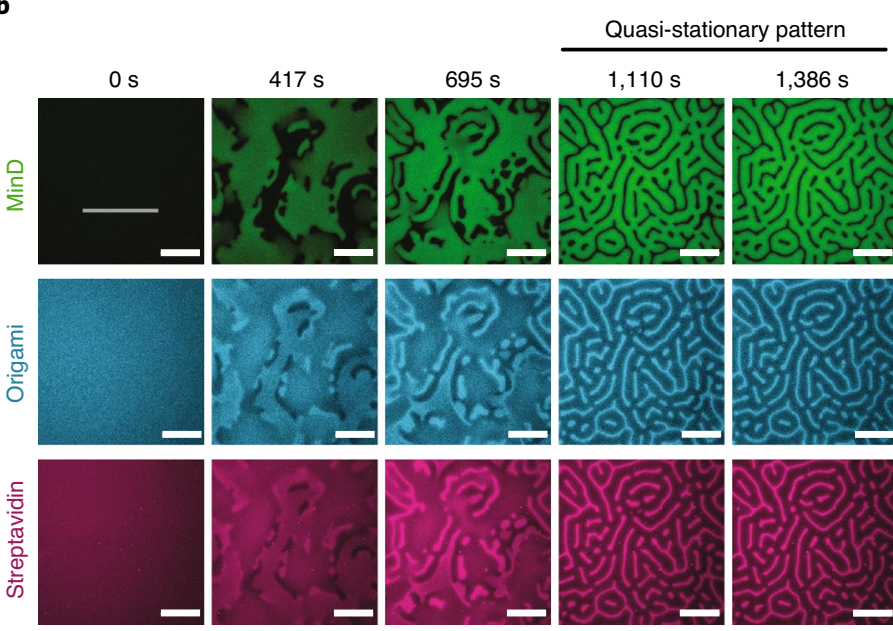

d
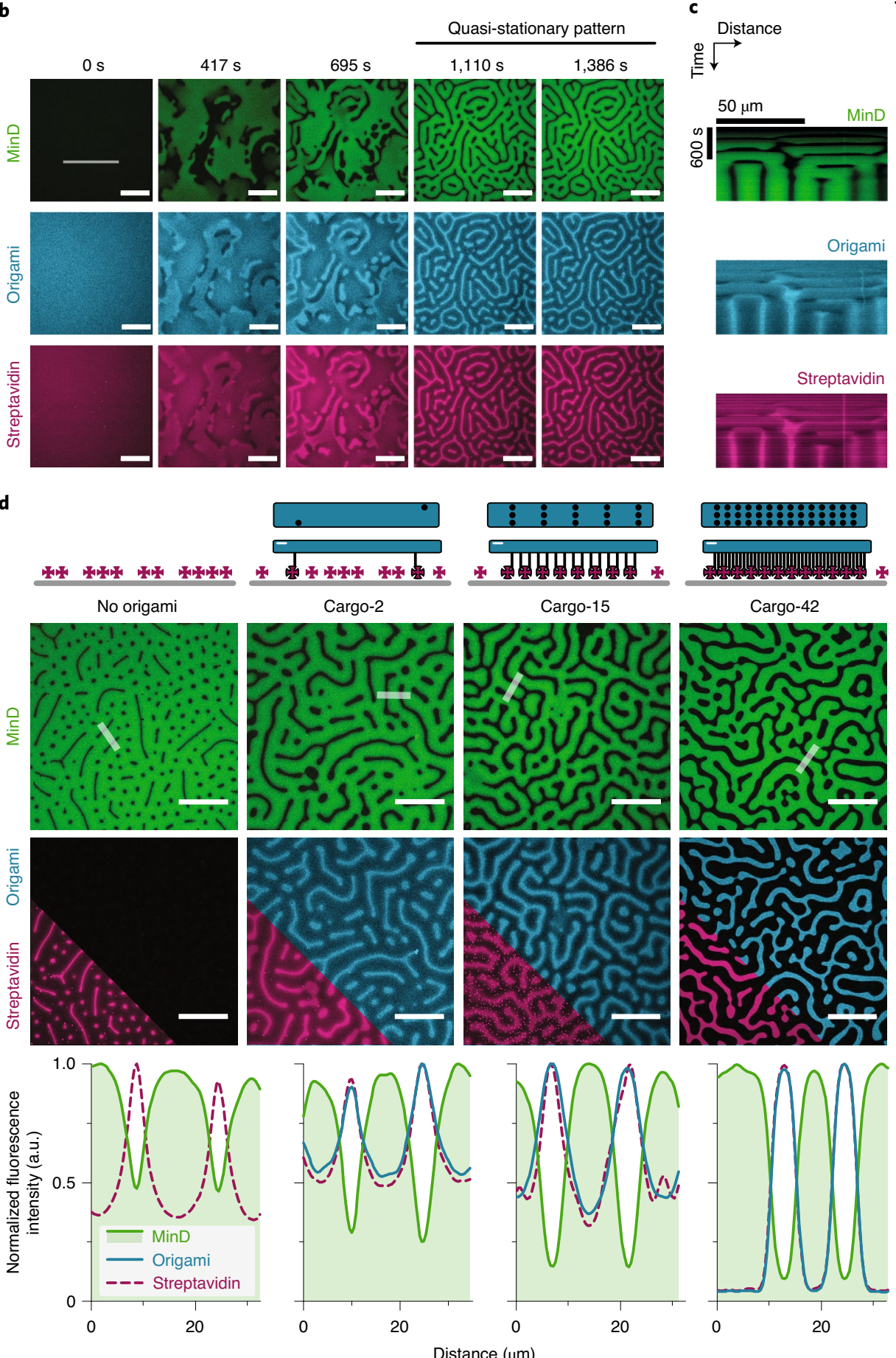

Origami

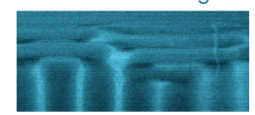

Cargo-42
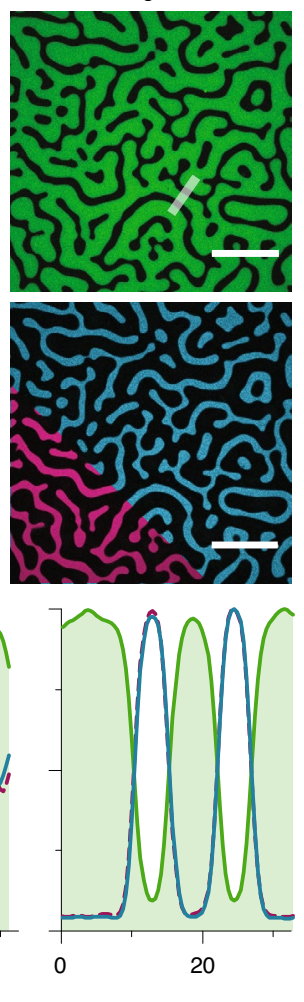

Biotinylated

$\begin{cases}\sim 7 \mathrm{~nm} & \text { oligonucleotides } \\ \tau \mathrm{T}^{2} & \text { Streptavidin/ }\end{cases}$ biotinylated lipids

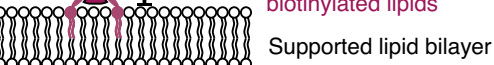
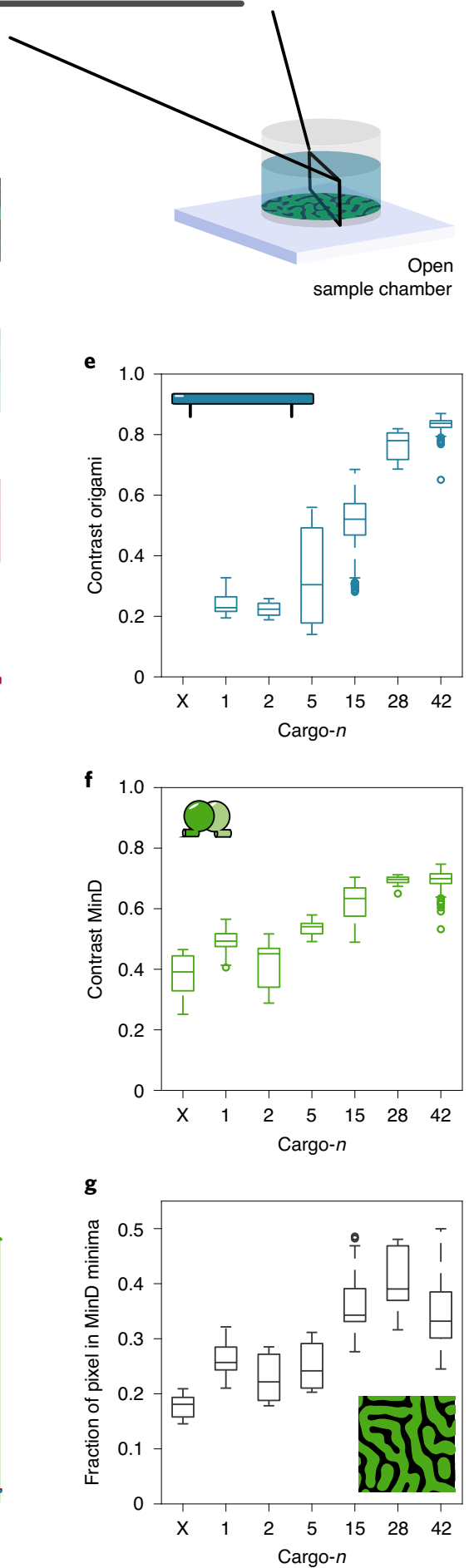

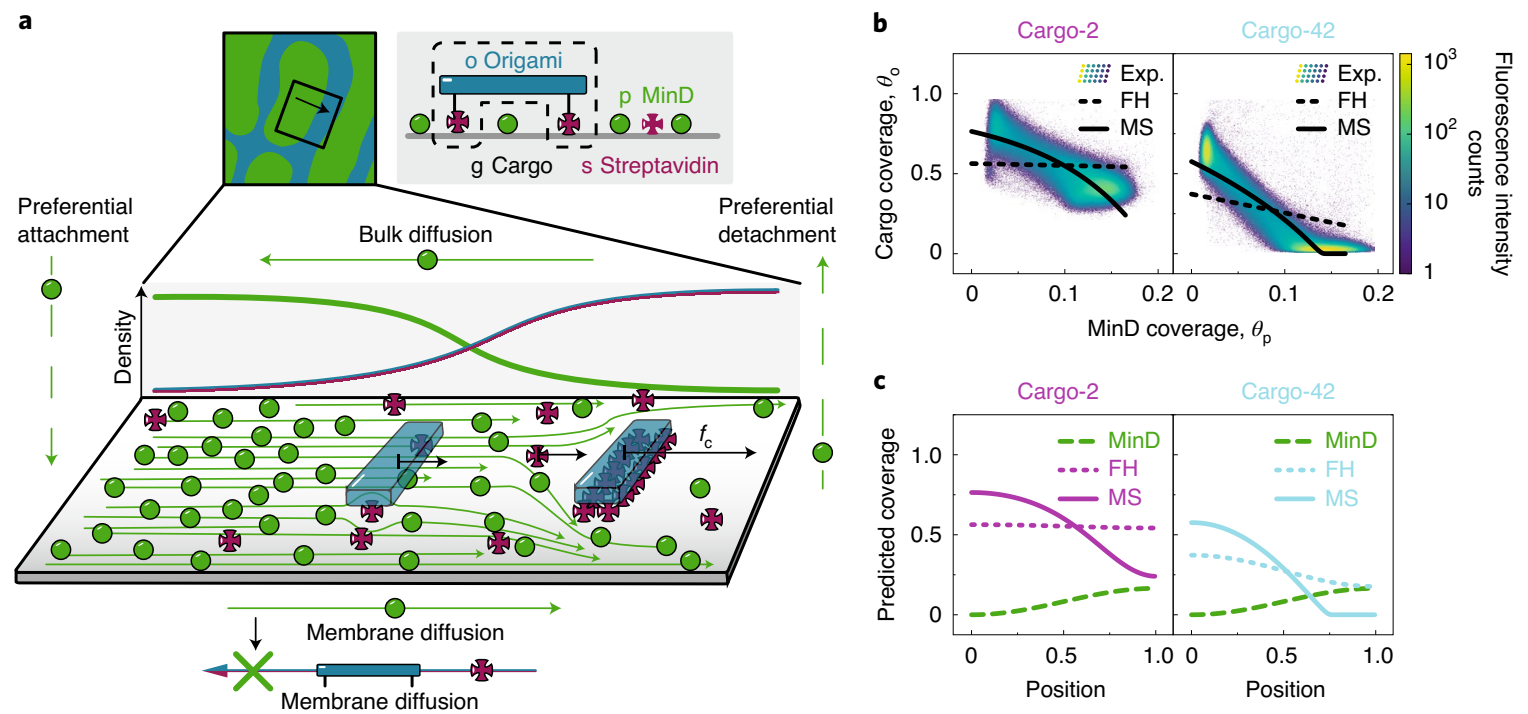

Fig. 2 | MinDE-dependent cargo transport is explained by mesoscopic friction between particle fluxes, not by mixing or volume exclusion. a, Schematic of the phenomenological MS-type model. MinD protein density gradients are determined by a closed cycle of reactive fluxes (MinD membrane attachment/detachment, dashed green arrows) and diffusive fluxes (green arrows). Mediated by hydrodynamic or direct interactions, the diffusive MinD membrane fluxes exert a frictional force $\mathbf{f}_{c^{\prime}}$ (black arrow) on the cargo molecules. Then, cargo density gradients are determined by a balance between these frictional forces and thermodynamic forces (entropic repulsion and mixing). b, Cross-correlation between MinD coverage $\left(\theta_{\mathrm{p}}=a_{\mathrm{p}} c_{\mathrm{p}}\right)$ and DNA origami coverage $\left(\theta_{0}=a_{0} c_{g}\right)$, for two different cargo species, cargo-2 and cargo-42. Respective membrane footprints: MinD dimer $a_{\mathrm{p}}=25 \mathrm{~nm}{ }^{2}$, DNA origami $a_{0}=1,760 \mathrm{~nm}^{2} \approx 70 a_{\mathrm{p}}$. The colour-coded two-dimensional histogram represents the experimental data of selected days (Exp.), where the raw images were smoothed by a one-pixel-wide Gaussian kernel. Solid and dashed lines correspond to two candidate models. The FH-type model (dashed lines), whose parameters are fully determined by our experiments, fails to account for cargo transport: weak entropic sorting of streptavidin in an external gradient of MinD is not sufficient to overcome the strong repulsion of the bulky DNA origami scaffolds. Instead, we find that the MS model (solid lines), with an estimated interaction parameter, explains cargo transport. The MS model allows stronger reorganization of cargo than the FH model, because, in addition to thermodynamic forces, cargo transport is further driven by frictional coupling to MinD protein fluxes. c, Spatial distribution of cargo molecules in response to the (imposed) MinD profile, corresponding to the cross-correlation functions in $\mathbf{b}$. Model parameters: (cargo-2) average coverages $\overline{\theta_{\mathrm{p}}}=0.0825, \overline{\theta_{\mathrm{s}}}=0.149$ and $\overline{\theta_{\mathrm{o}}}=0.55$; interaction parameter in terms of MinD coverage $\theta_{\times}^{-1}=a_{\mathrm{p}}^{-1} c_{\times}^{-1}=220$; (cargo-42) average coverages $\overline{\theta_{\mathrm{p}}}=0.0825, \overline{\theta_{\mathrm{s}}}=0$ and $\overline{\theta_{\mathrm{o}}}=0.28$; interaction parameter $\theta_{\times}^{-1}=a_{\mathrm{p}}^{-1} c_{\times}^{-1}=620$.

gradient of passive cargo in response to a gradient of active MinD, where the cargoes' crosslinked streptavidin experience a weak entropic bias towards MinD-depleted regions (due to volume exclusion effects). However, this effect will be partly compensated by the analogous entropic repulsion between origami scaffolds, which prevents high cargo densities, in stark contrast to our experimental observations (Fig. 2b,c and Extended Data Fig. 3). Consequently, we also rejected entropic mixing in fixed external (chemical potential) gradients as the mechanism underlying MinDE-dependent cargo transport.

\section{Coupling between particle fluxes explains cargo transport}

Taken together, thermodynamic mechanisms alone cannot explain MinDE-induced cargo transport in our experiments, suggesting that the underlying mechanism is genuinely non-equilibrium in nature. Therefore, we generalized our equilibrium FH model to consider the non-equilibrium dynamics of particles. Specifically, MinDE gradients build up due to particle exchange between the bulk solution and membrane (reactive fluxes) ${ }^{16,18,19,21,25}$, leading to chemical potential gradients $\nabla \mu_{i}$ on the membrane (thermodynamic forces). According to Onsager's theory ${ }^{34}$, thermodynamic forces imply particle fluxes $\mathbf{j}_{i}$. Conceptually, diffusive fluxes on the membrane then arise from an effective force balance of thermodynamic and friction forces between the different macromolecules and lipids (with friction coefficients $\xi_{i}$ ). Furthermore, in a crowded environment such as the membrane surface in our experiments (mean free path of $\sim 50 \AA$ A S Supplementary Information), hydrodynamic or also direct interactions between particles can mediate a 'mesoscopic friction' that couples their respective fluxes (with coupling constants $\zeta_{i k}$ ). Using these arguments, we formulated a phenomenological theory where each membrane-bound species obeys a Maxwell-Stefan (MS)-like effective force-balance equation ${ }^{35,36}$ :

$$
\nabla \mu_{i}+\xi_{i} \frac{\mathbf{j}_{i}}{c_{i}}+\sum_{k} c_{k} \zeta_{i k}\left(\frac{\mathbf{j}_{i}}{c_{i}}-\frac{\mathbf{j}_{k}}{c_{k}}\right)=0
$$

As before, the index $i \in\{p, g, s\}$ refers to MinD proteins, cargo with bound streptavidin and free streptavidin, respectively. Although our theory is intrinsically dynamic (Methods), we first analysed the non-equilibrium steady state, $\partial_{t} c_{i}=0$, reflecting the quasi-stationary MinDE patterns. Then, cargo and streptavidin exhibit Brownian motion and relax to a thermal equilibrium state with vanishing fluxes $\mathbf{j}_{\mathrm{g}}=\mathbf{j}_{\mathrm{s}}=0$. The MinD protein patterns maintain a non-equilibrium steady state and finite protein fluxes $\mathbf{j}_{\mathrm{p}} \neq 0$ via off-equilibrium chemical reactions (ATPase activity). In the absence of mutual friction between the macromolecules $\left(\zeta_{i k}=0\right)$, equation (1) reduces to the FH model $\left(\nabla \mu_{\mathrm{g}}=\nabla \mu_{\mathrm{s}}=0\right)$, which readily implies weak cargo redistribution in a static gradient of active proteins. As a decisive factor in addition to these entropic demixing effects, the presence of frictional coupling $\left(\zeta_{i k} \neq 0\right)$ between cargo and MinD leads to advective cargo transport by the non-equilibrium protein fluxes $\left(\mathbf{j}_{\mathrm{p}} \neq 0\right)$ of MinD (Fig. $\left.2 \mathrm{a}\right)$. The additional bias conferred by these protein fluxes leads to cargo redistribution, which is much stronger than by equilibrium thermodynamic forces alone and thus quantitatively explains our experimental data (Fig. 2b,c and Extended Data Fig. 3). 
a

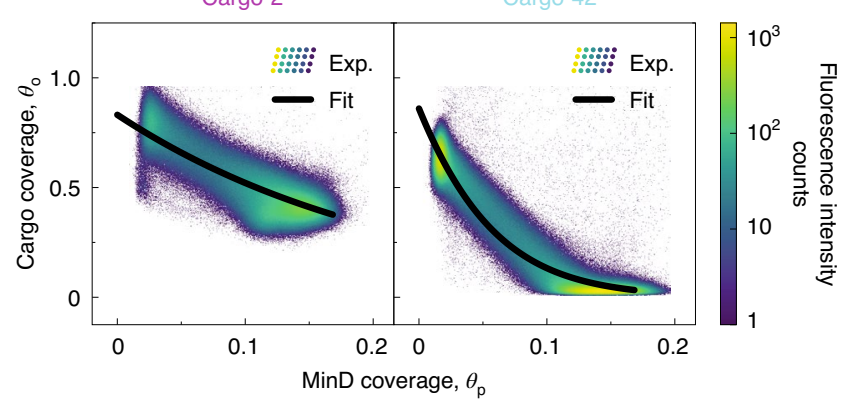

b

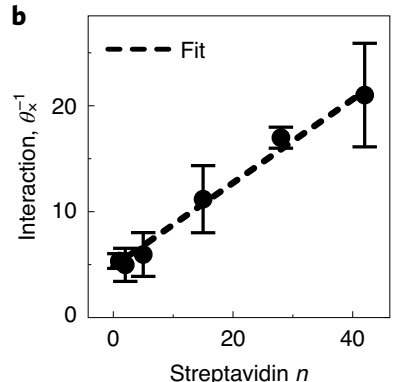

c

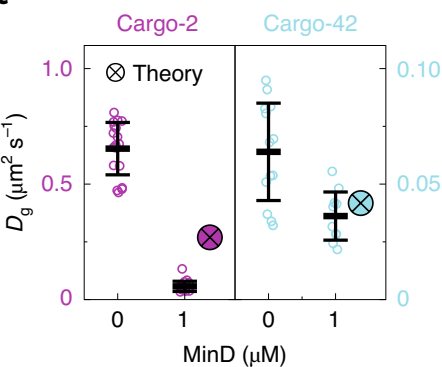

Fig. 3 | Reduced model predicts that large MinD densities slow down cargo diffusion. a, Cross-correlation between MinD coverage $\left(\theta_{p}=a_{p} c_{p}\right)$ and DNA origami coverage $\left(\theta_{0}=a_{0} c_{g}\right)$ for cargo- 2 and cargo42. Respective membrane footprints: MinD dimer $a_{\mathrm{p}}=25 \mathrm{~nm}^{2}$, DNA origami $a_{0}=1,760 \mathrm{~nm}^{2}$. The colour-coded two-dimensional histogram represents experimental data for selected days (Exp.), identical to Fig. 2b, while the solid line (Fit) is a fit curve of our reduced model. b, Interaction parameter in terms of MinD coverage, $\theta_{\times}^{-1}=a_{\mathrm{p}}^{-1} c_{\times}^{-1}$, obtained by the fitting procedure in $\mathbf{a}$. The interaction linearly increases when more streptavidin is incorporated per cargo, starting from the base interaction of the origami scaffold. The dashed line indicates a linear fit of the interaction parameter $\theta_{\times}^{-1}=\left(\zeta_{p o}+n \zeta_{p s}\right) /\left(a_{p} \xi_{p}\right)$ as a function of $n$. In contrast to our initial estimate (Fig. 2c), entropic repulsion between cargo molecules was neglected for simplicity, thus probably underestimating the interaction parameter. c, Cargo molecule diffusion coefficient in the absence or presence of $1 \mu \mathrm{M} \mathrm{MinD}$ ( $1 \mu \mathrm{M}$ MinD (30\% EGFP-MinD), 0.1-1 pM origami-Cy3b with 2 or 42 biotinylated oligonucleotides, non-labelled streptavidin, SLB: DOPC/ DOPG/biotinyl-CAP-PE 70/30/0.01 mol\%), measured via single-particle tracking and predicted with the fitted interaction parameters from $\mathbf{b}$. Cargo-42 typically diffuses slower than cargo-2 (indicated by a smaller diffusion coefficient $D_{g}$ at $0 \mu \mathrm{M}$ MinD). Increasing MinD density has a much stronger effect on cargo-2 than on cargo-42, both in theory and experiment. Points are mean values of individual measurements $(M)$, line and error bars represent the mean value and standard deviation. Data were obtained from the number of sample chambers $S($ Cargo- 2$)=7$, $S($ Cargo-42) $=5, S($ Cargo-2, MinD $)=4, S($ Cargo-42, MinD $)=3$; number of measurements $M($ Cargo- 2$)=18, M($ Cargo-42 $)=13, M($ Cargo-2,MinD $)=19$, $M($ Cargo-42, MinD $)=10$; number of analysed single-particle tracks $N($ Cargo- $)=15,755, N($ Cargo-42 $)=19,481, N($ Cargo $-2, M i n D)=7,924$, $N($ Cargo-42, MinD $)=4,542$; average track length $T L($ Cargo-2 $)=339$, $\mathrm{TL}($ Cargo-42) $=546, \mathrm{TL}($ Cargo-2, MinD $)=772, \mathrm{TL}($ Cargo $-42, \mathrm{MinD})=647$; fraction of mobile DNA origami $M F($ Cargo- 2$)=0.81, M F($ Cargo- 42$)=0.67$, $\mathrm{MF}($ Cargo-2, MinD $)=0.70, \mathrm{MF}($ Cargo-42, MinD $)=0.63$.

\section{MinDE impair cargo diffusion via mesoscopic friction}

Next, we simplified our theoretical model by neglecting membrane saturation effects (Supplementary Information), reducing the chemical potential of particles with size $a_{i}$ to $\mu_{i} \approx k_{\mathrm{B}} T \ln \left(a_{i} c_{i}\right)$. The force-balance equation, equation (1), then becomes a generalized Fick's law:

$$
\mathbf{j}_{\mathrm{p}} \approx-\frac{k_{\mathrm{B}} T}{\xi_{\mathrm{p}}+c_{\mathrm{g}} \zeta_{\mathrm{pg}}+c_{\mathrm{s}} \zeta_{\mathrm{ps}}} \nabla c_{\mathrm{p}} \equiv-D_{\mathrm{p}}\left(c_{\mathrm{g}}, c_{\mathrm{s}}\right) \nabla c_{\mathrm{p}}
$$

where the density-dependent diffusion coefficient of MinD, $D_{\mathrm{p}}\left(c_{\mathrm{g}}, c_{\mathrm{s}}\right)$, decreases through friction (that is, interactions) with cargo and streptavidin. For cargo with strong coupling $\zeta_{\mathrm{pg}}$, maintaining the diffusive fluxes that balance reactive protein turnover requires sharper protein gradients, which explains the progressively sharper and narrower MinDE patterns (Fig. 1f,g).

Assuming that the typically small number of free streptavidin (Supplementary Information) does not notably contribute to the dynamics, $c_{\mathrm{s}} \zeta_{\mathrm{ps}} \ll c_{\mathrm{g}} \zeta_{\mathrm{pg}}$, one obtains a closed expression for the cargo distribution:

$$
c_{\mathrm{g}}\left(c_{\mathrm{p}}\right)=c_{\times} W_{0}\left[\frac{c_{\mathrm{g}}(0)}{c_{\times}} \exp \left(\frac{c_{\mathrm{g}}(0)-c_{\mathrm{p}}}{c_{\times}}\right)\right]
$$

with typical interaction density $c_{\times}=\xi_{\mathrm{p}} / \zeta_{\mathrm{pg}}$. Fitting equation (3) to our experimental data (Fig. 3a,b and Extended Data Fig. 3), we demonstrate that the coupling constant $\zeta_{\mathrm{pg}}$ between MinD and cargo- $n$ has a contribution from the origami scaffold and from the $n$ incorporated streptavidin, $\zeta_{\mathrm{pg}}=\zeta_{\mathrm{po}}+n \zeta_{\mathrm{ps}}$, explaining why cargo transport increases with the number of streptavidin per cargo (Fig. 1d-g).

To test our theoretical model experimentally, we performed single-particle tracking of cargo- 2 and cargo- 42 , both in the presence and absence of MinD. In the former case, we emulated high MinD membrane densities in the maxima of MinDE patterns by adding $1 \mu \mathrm{M}$ MinD and ATP, but no MinE. We found that the diffusion coefficient of cargo-2 decreased from $0.65 \pm 0.12 \mu \mathrm{m}^{2} \mathrm{~s}^{-1}$ in the absence of MinD to $0.06 \pm 0.02 \mu \mathrm{m}^{2} \mathrm{~s}^{-1}$ at high MinD density (Fig. 3c). By contrast, the diffusion coefficient of cargo-42, which was already low in the absence of $\operatorname{MinD}\left(0.06 \pm 0.02 \mu \mathrm{m}^{2} \mathrm{~s}^{-1}\right)$, hardly changed at high MinD density $\left(0.036 \pm 0.011 \mu \mathrm{m}^{2} \mathrm{~s}^{-1}\right)$ (Fig. $3 c$ ). Subsequently, we used our fitted interaction parameters to predict the diffusion coefficient of cargo at high protein densities, based on the experimental values in the absence of proteins (Supplementary Information). Our predictions were in good quantitative agreement with our experimental findings, validating our model. At first, it might seem counterintuitive that MinD affects cargo-42 diffusion less than that of cargo-2, despite stronger frictional coupling. However, even in the absence of MinD, cargo-42 diffuses much slower than cargo-2 due to the friction between its many streptavidin and the membrane, which dominates over the additional friction with MinD. This observation highlighted the parameter interdependence that arises due to streptavidin serving both as building block and connector to the membrane and raised the question whether cargo transport is affected more by its effective size or its diffusion coefficient. To answer this, we employed an alternative membrane attachment strategy for the DNA origami scaffolds via cholesteryl moieties (Extended Data Figs. 4 and 5 and Supplementary Note 2). We found that cargoes with a larger membrane footprint (that is, effective size) and at the same time similar diffusion as cargo-2 were strongly redistributed (Extended Data Fig. 5 and Supplementary Note 2), corroborating that it is indeed the effective cargo size that determines the interaction with MinD. In conclusion, the dependence of the cargo diffusion coefficient on the ambient protein density is a direct experimental proof of friction between MinD and cargo and that the effective cargo size governs the corresponding friction coefficient.

\section{MinDE spatially sort different cargo species}

Can we use our obtained knowledge to selectively position cargo molecules, that is, to sort them according to their properties, along protein gradients? To answer this question, we placed two differently labelled cargo species, cargo- 2 and cargo- 42 , in the same assay (Fig. 4a). We found, as predicted by our model (Fig. 4d), that cargo- 
a

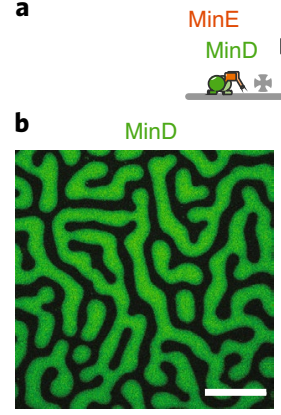

C

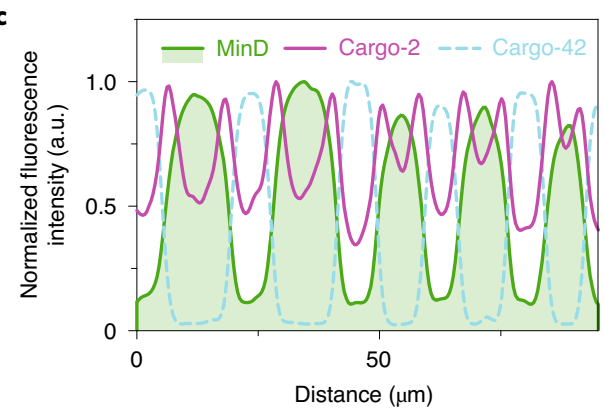

Cargo-2 with Cy3B

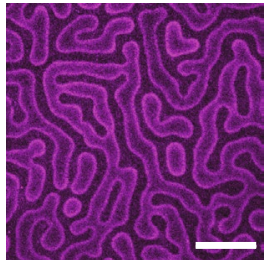

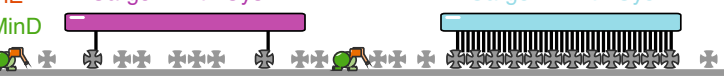

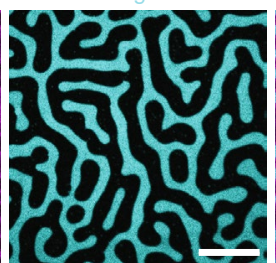

Cargo-2/C

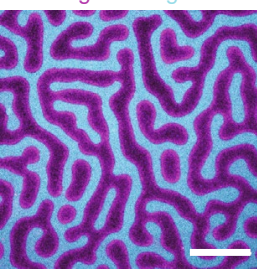

d

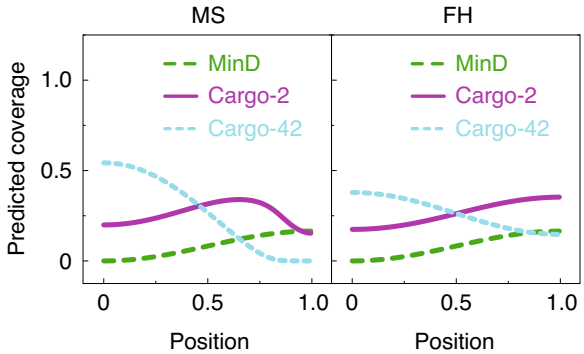

Fig. 4 | MinDE spatially sort cargo according to effective size. a, Schematic of the experimental set-up. MinDE self-organization was performed in the presence of two different cargo species with distinct fluorescent labels, cargo- 2 with Cy3B and cargo-42 with Cy5 (1 $\mu$ M MinD (30\% EGFP-MinD), $1.5 \mu$ M MinE-His, 50 pM origami-Cy3b with two biotinylated oligonucleotides, and 50 pM origami-Cy 5 with 42 biotinylated oligonucleotides, non-labelled streptavidin). b,c, Representative images of individual and overlaid channels (b) and line plot (c) (smoothed) of the indicated selection of MinDE-induced sorting of cargo species. Scale bars, $50 \mu \mathrm{m}$. The experiment was performed three times under identical conditions. $\mathbf{d}$, Spatial distribution of two cargo species in response to the (imposed) MinD profile. The phenomenological MS-type model allows for stronger reorganization of cargo molecules than the FH-type model. In particular, the phenomenological MS-type model predicts that cargo- 2 accumulates between cargo-42 and MinD. Model parameters: average coverage of MinD proteins $\overline{\theta_{\mathrm{p}}}=0.0825$, streptavidin $\overline{\theta_{\mathrm{s}}}=0$, cargo- $2 \overline{\theta_{0}-2}=0.26$ and cargo-42 $\overline{\theta_{0}-42}=0.26$; interaction parameter (in terms of MinD coverage: $\theta_{\times}^{-1}=a_{\mathrm{p}}^{-1} c_{\times}^{-1}$ ) of cargo- $2 \theta_{\times-2}^{-1}=220$ and cargo-42 $\theta_{\times-42}^{-1}=620$. Respective membrane footprints: MinD dimer $a_{\mathrm{p}}=25 \mathrm{~nm}{ }^{2}$, streptavidin $a_{\mathrm{s}}=25 \mathrm{~nm}^{2}$, DNA origami $a_{\mathrm{o}}=1,760 \mathrm{~nm}^{2} \approx 70 a_{\mathrm{p}}$.

42 gathered in MinD-free regions and was framed by cargo-2 (Fig. 4b,c, Supplementary Fig. 3 and Supplementary Video 3). Thus, cargo- 42 exhibited a similar behaviour as when present in the assay alone. Bycontrast, thelocalization of cargo-2 relative to MinD changed when cargo-42 was also present (Figs. $1 \mathrm{~d}$ and $4 \mathrm{c}$ ). The observed spatial separation of cargo species was not an artefact due to fluorescent channel crosstalk, dye selection or quenching (Extended Data Fig. 6). Hence, the clear MinDE-induced spatial sorting of cargo species according to their effective size further refutes thermodynamic models (Fig. 4d), corroborating that MinDE indeed transport molecules via friction.

\section{MinDE waves direct net cargo transport via diffusiophoresis}

Having confirmed diffusiophoretic transport in the context of quasi-stationary MinDE patterns, we turned to conditions where MinDE form travelling surface waves $^{7,8}$. As in previous experiments with lipid-anchored streptavidin alone ${ }^{7,8}$, MinDE waves induced anti-correlated patterns of our synthetic cargo-2 that were superimposed with macroscopic gradients across multiple MinDE wavelengths, indicating net transport (Fig. 5a). Consequently, cargo accumulated between opposing wavefronts (Fig. 5a and Supplementary Video 4). Despite their distinct appearance, stationary as well as moving MinDE patterns are maintained by the same kind of non-equilibrium reaction and diffusion fluxes ${ }^{16,18,19,21,25}$. Thus, we could directly apply our theory to this dynamic setting, without further assumptions (Methods). We found that, in addition to the interaction between MinDE and cargo, macroscopic transport also requires matching timescales between MinDE wave propagation and cargo diffusion: cargo with small mobility cannot keep up with the MinDE wave. In agreement with our simulations (Fig. 5b), the slowly diffusing cargo-42 hardly formed long-ranged gradients (Fig. 5c and Supplementary Fig. 4), despite its strong frictional coupling to MinDE, supporting our prediction that low cargo mobility can limit macroscopic transport. This was further corroborated by the emergence of macroscopic gradients for cholesterol-anchored cargo-chol-2 and cargo-chol-15 (Fig. 5c and Supplementary Fig. 4), which display similarly high coupling to MinDE, but diffuse faster than cargo-42 (Extended Data Fig. 5).

Next, we exploited the geometry-sensitivity of MinDE waves that travel along the longest axis on geometrically patterned planar membranes with chromium barriers ${ }^{37}$. On these membranes, MinDE waves indeed transported cargo directionally along their wavevector, resulting in reproducible gradients (Fig. 5d,e, Extended Data Fig. 7 and Supplementary Videos 5 and 6). These density gradients are clearly visible on the macroscopic scale, while directed movement of single cargo molecules cannot be discerned from diffusion (Extended Data Fig. 8 and Supplementary Note 3). This observation highlights the difference between ATP-driven diffusiophoresis and translational motor proteins.

Our findings raised the question of whether MinDE-dependent transport via diffusiophoresis could occur in vivo. To our knowledge, no direct observation of this mechanism in $E$. coli has been reported, which may be attributed to bacterial sizes close to the optical resolution limit or specific interactions concealing such occurrence. Thus, we reconstituted MinDE oscillations together with inert model peripheral membrane proteins (mCh-MTS ${ }^{7}$ in the evolutionary distant fission yeast Schizosaccharomyces pombe $e^{38}$ 


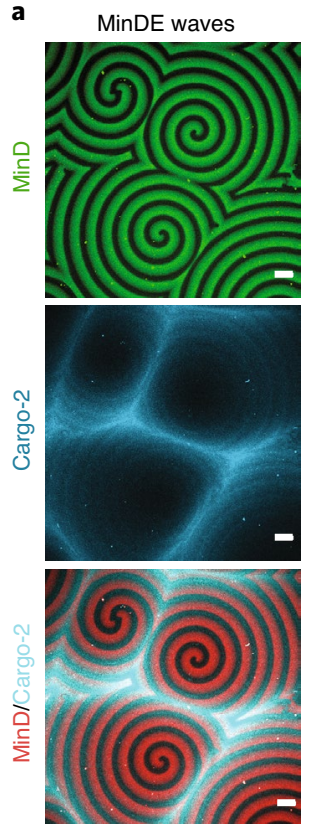

f

Reconstitution in vivo b

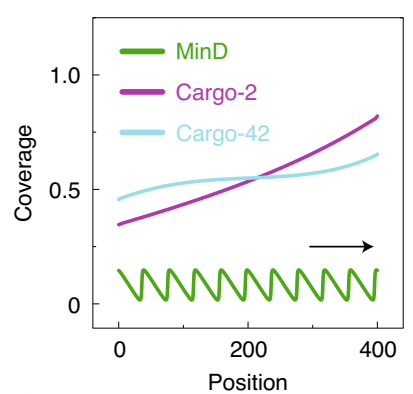

d

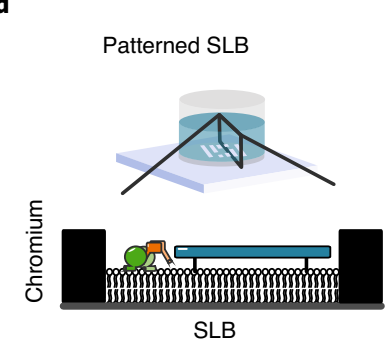

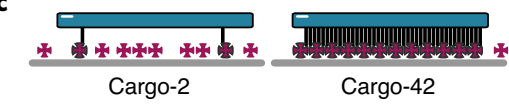
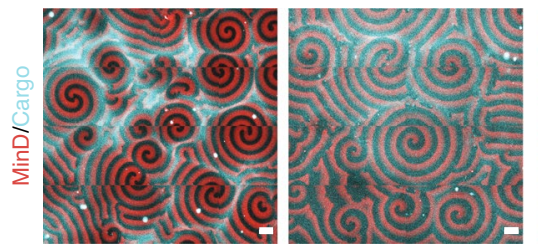
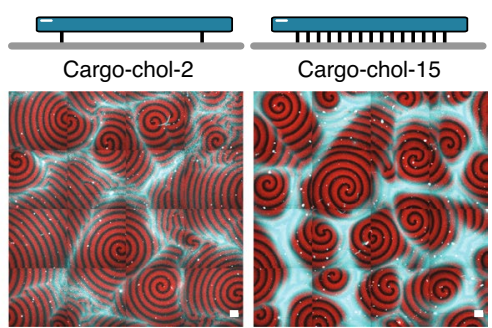
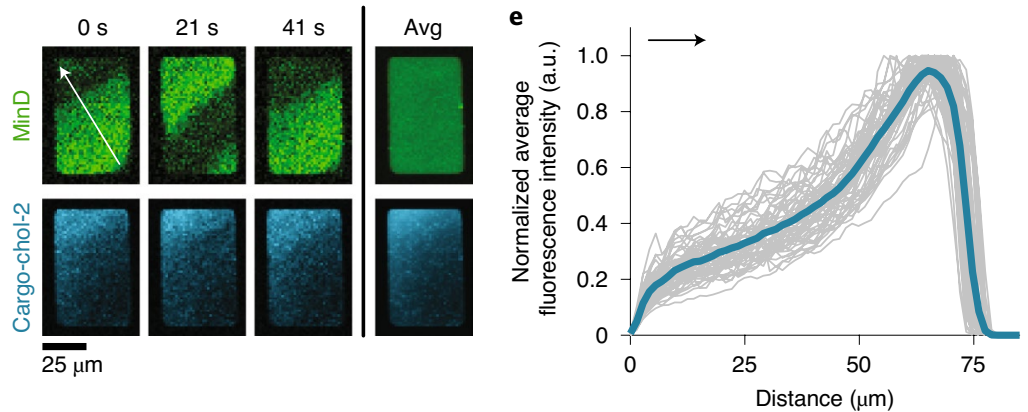

$30 \mathrm{~s}$
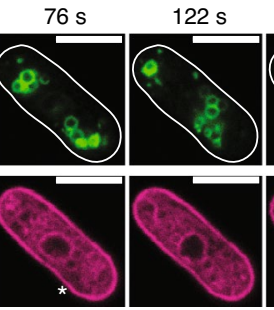

$186 \mathrm{~s}$
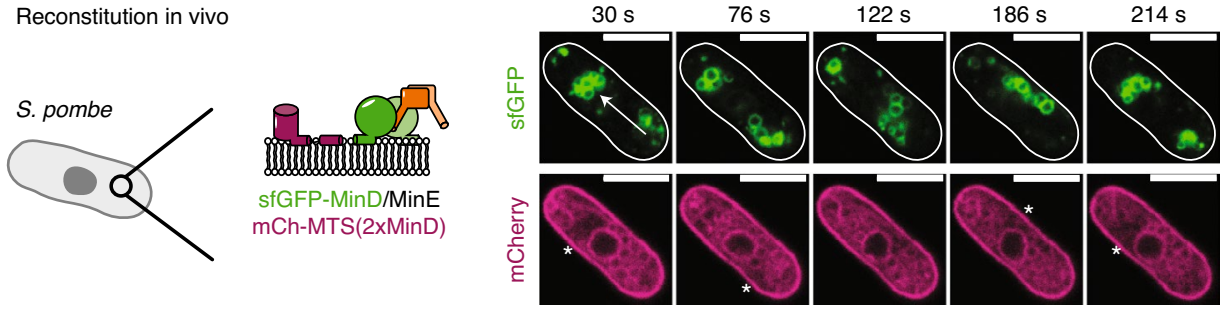

mCh-MTS(2xMinD)
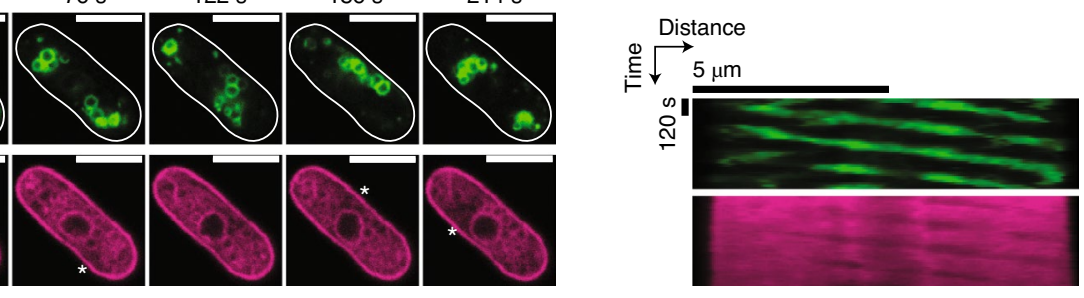

Fig. 5 | Directed net transport of cargo by MinDE waves via diffusiophoresis. a, Representative images of MinDE travelling surface waves that transport cargo-2 establishing long-range gradients ( $1 \mu \mathrm{M}$ MinD, $5 \mu \mathrm{M}$ His-MinE, $0.1 \mathrm{nM}$ origami-Cy3b with two biotinylated oligonucleotides, non-labelled streptavidin). b. Simulation of cargo transport by a protein wave with skewed sinusoidal waveform (green, skewness $\gamma=-0.9$ ), which emulates MinD travelling waves ( $40 \mu \mathrm{m}$ wavelength, $1.4 \mu \mathrm{m} \mathrm{s}^{-1}$ velocity) in one-dimensional geometry (the black arrow indicates the wavevector). After a very long simulation time of $28 \mathrm{~h}$, we find only weak redistribution of cargo- 42 compared with the strong redistribution of cargo-2. Interaction parameters are as in Fig. 3b, (bare) cargo diffusion coefficients $D_{g}(0)$ as in Fig. $3 c$ and the (bare) MinD diffusion coefficient is set to $D_{p}=0.425 \mu m^{2} s^{-1}$ (ref. ${ }^{25}$ ). Both cargo coverages are set to $\overline{\theta_{0}}=0.55$ (thus neglecting the reduced surface coverage of cargo-42) to improve comparability. Cargo coverages are averaged over the last $17 \mathrm{~min}$. c, Representative images of MinDE inducing large-scale gradients of cargo-2, cargo-chol-2 and cargo-chol-15, but not of cargo-42 $(1 \mu \mathrm{M}$ MinD, $5 \mu \mathrm{M}$ His-MinE, $0.1 \mathrm{nM}$ origami-Cy 5 with 2 or 42 biotinylated oligonucleotides, non-labelled streptavidin or $0.1 \mathrm{nM}$ origami-Cy3b with 2 or 15 hybridizing oligonucleotides, $10 \mathrm{nM}$ TEG-cholesteryl oligonucleotide). d, Representative time series and average of MinDE travelling surface waves transporting cargo-chol-2 along the wavevector when oriented along the longest axis on chromium-patterned SLBs e, Normalized average fluorescence intensity profiles of cargo along the wavevector in $\mathbf{d}$. The bold coloured line represents the mean profile, generated from $N=56$ membrane patches from three independent experiments. f, MinDE dynamics spatiotemporally regulates the model peripheral membrane proteins mCh-MTS(2xMinD) when reconstituted in the fission yeast S. pombe. Decreased fluorescence in the mCherry channel is highlighted by white asterisks. Arrows indicate wave direction. Scale bars, $50 \mu \mathrm{m}(\mathbf{a}, \mathbf{c}), 25 \mu \mathrm{m}$ (d) and $5 \mu \mathrm{m}$ (f).

(Fig. 5f, Extended Data Figs. 9 and 10 and Supplementary Videos 7 and 8). We found that in this in vivo model system MinDE formed dynamic patterns with similar wavelength and velocity as in its native host E. coli ${ }^{11,12}$ (Supplementary Note 4) and, indeed, spatiotemporally regulated mCh-MTS proteins on intracellular membranes (Extended Data Fig. 10).

\section{Diffusiophoresis drives molecular transport in biology}

In conclusion, combining in vitro and in vivo reconstitution experiments with theoretical modelling and analysis, we showed that the prokaryotic MinDE proteins can non-specifically transport and even sort membrane-bound cargo molecules by a diffusiophoretic effect: the diffusive fluxes of MinD and cargo couple via friction in a mechanical rather than thermodynamic fashion (Fig. 6). This process is driven by ATP-consuming MinDE self-organization, which generates a net diffusive flux of MinD towards low densities. This flux establishes an effective frictional force on cargo, driving diffusiophoretic cargo transport towards areas of low MinD density. Hence, the friction between cargo and MinD increases with the effective size of the cargo (for example, with the number of streptavidin building blocks). The bare diffusion coefficient of cargo only becomes relevant in the case of dynamic MinDE waves where cargo diffusion has to 'keep up' with the MinDE waves to induce macroscopic gradients.

Similar transport effects have been reported previously in a non-biological context: diffusiophoresis generally refers to particle transport in fluids, induced by concentration gradients of small solutes $^{39-49}$. Interestingly, diffusiophoresis was also treated in terms of particle fluxes ${ }^{48}$, suggesting that a flux-centred viewpoint may be appropriate for multicomponent mixtures out of equilibrium. 

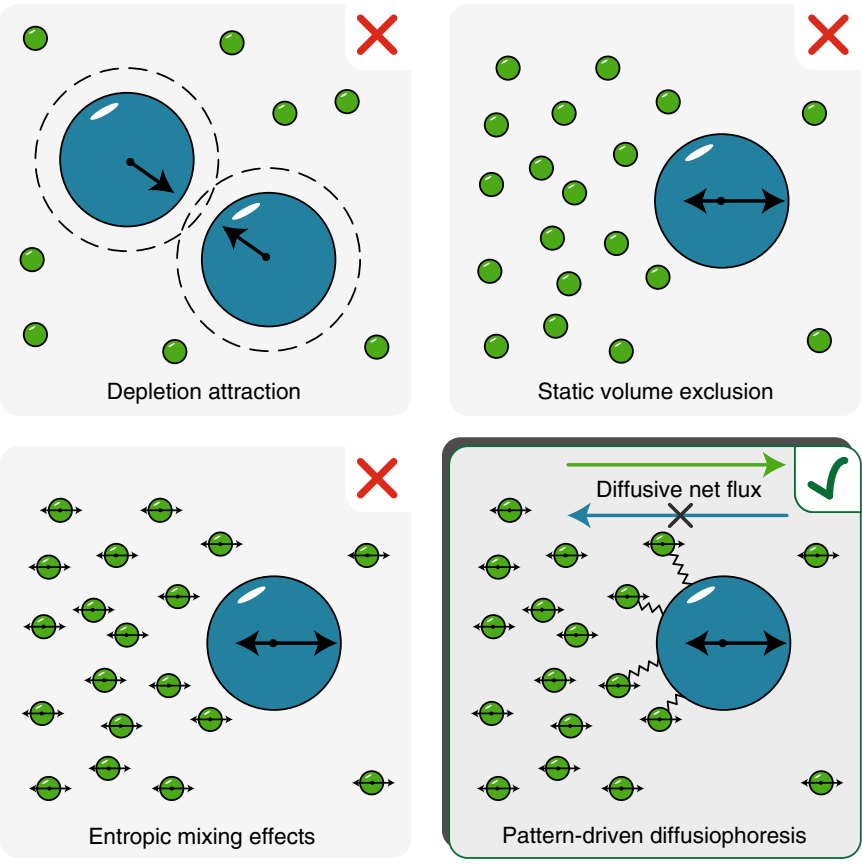

Fig. 6 | Diffusiophoresis explains cargo transport by protein self-organization. Thermodynamic forces, such as depletion attraction, static volume exclusion or entropic mixing effects, cannot explain MinDE-dependent cargo transport in our experiments. However, it can be explained by diffusiophoresis: active protein self-organization generates gradients and hence net diffusive fluxes. As the proteins interact non-specifically with the cargo, the diffusive fluxes establish an effective frictional force, driving the accumulation of cargo in areas of low protein density.

A phenomenologically similar effect has been demonstrated in gases with the MS theory of diffusion ${ }^{35,36,50,51}$. In a biologically relevant context, theoretical studies have suggested that diffusiophoresis might occur in enzyme chemotaxis ${ }^{52}$ and alignment ${ }^{53}$, during ParABS-mediated chromosome segregation in bacteria ${ }^{54}$ and that metabolism-dependent gradients of small molecules (for example, ATP) could induce transport of large particles ${ }^{55}$. The here described diffusiophoresis driven by protein patterns that are maintained by NTPase cycles might constitute a novel mechanism of coupling energy dissipation to active spatiotemporal positioning in cell biology. Diffusiophoretic transport is presumably not a special feature of the E. coli MinDE system or reaction-diffusion systems in general, but can potentially be exerted by any active system producing concentration gradients. For example, such a mechanism could be underlying the secretion-induced protein patterning that has been observed in fission yeast ${ }^{56}$ or be at play for the plethora of intracellular (actin) waves in eukaryotes whose purpose and mode of action has remained elusive ${ }^{57}$. The mechanism might not even be limited to the membrane as a reaction surface, but potentially extends to other cellular surfaces and even cytosolic gradients. For example, the strong concentration gradients that are built up during liquidliquid phase-separation processes could potentially similarly impact other molecules ${ }^{58}$. We also propose that distinct pattern-forming systems sharing the same reaction space should align to minimize friction, even if their constituents are chemically independent. This could potentially link and synchronize pathways to increase their efficacy or provide a rescue mechanism against mutations affecting the chemical coupling via specific interactions (for example, between MinC and FtsZ). That this non-specific means of transport was discovered and described in an in vitro reconstitution assay is not a coincidence, but highlights that the complexity of cells with more sophisticated and stronger specific interactions presumably masks such occurrence. Finally, simple as it is in comparison to eukaryotic, translational motor proteins, this mechanism could be interpreted as an alternative, more rudimentary mode of mechanochemical coupling and as such might be prevalent in prokaryotes and might have been present in early forms of life.

\section{Online content}

Any methods, additional references, Nature Research reporting summaries, source data, extended data, supplementary information, acknowledgements, peer review information; details of author contributions and competing interests; and statements of data and code availability are available at https://doi.org/10.1038/ s41567-021-01213-3.

Received: 1 May 2020; Accepted: 22 February 2021;

Published online: 5 April 2021

\section{References}

1. Vale, R. D. The molecular motor toolbox for intracellular transport. Cell 112, 467-480 (2003)

2. Theriot, J. A. The polymerization motor. Traffic 1, 19-28 (2000).

3. Halatek, J., Brauns, F. \& Frey, E. Self-organization principles of intracellular pattern formation. Phil. Trans. R. Soc. B 373, 20170107 (2018).

4. Almonacid, M. et al. Active diffusion positions the nucleus in mouse oocytes. Nat. Cell Biol. 17, 470-479 (2015).

5. Colin, A. et al. Active diffusion in oocytes nonspecifically centers large objects during prophase I and meiosis I. J. Cell Biol. 219, e201908195 (2020).

6. Goehring, N. W. et al. Polarization of PAR proteins by advective triggering of a pattern-forming system. Science 334, 1137-1141 (2011).

7. Ramm, B. et al. The MinDE system is a generic spatial cue for membrane protein distribution in vitro. Nat. Commun. 9, 3942 (2018).

8. Shih, Y.-L. et al. Active transport of membrane components by self-organization of the Min proteins. Biophys. J. 116, 1469-1482 (2019).

9. de Boer, P. A. J., Crossley, R. E. \& Rothfield, L. I. A division inhibitor and a topological specificity factor coded for by the minicell locus determine proper placement of the division septum in E. coli. Cell 56, 641-649 (1989).

10. Hu, Z. \& Lutkenhaus, J. Topological regulation of cell division in Escherichia coli involves rapid pole to pole oscillation of the division inhibitor MinC under the control of MinD and MinE. Mol. Microbiol. 34, 82-90 (1999).

11. Raskin, D. M. \& de Boer, P. A. J. Rapid pole-to-pole oscillation of a protein required for directing division to the middle of Escherichia coli. Proc. Natl Acad. Sci. USA 96, 4971-4976 (1999).

12. Ramm, B., Heermann, T. \& Schwille, P. The E. coli MinCDE system in the regulation of protein patterns and gradients. Cell. Mol. Life Sci. 76, 4245-4273 (2019).

13. Loose, M., Fischer-Friedrich, E., Ries, J., Kruse, K. \& Schwille, P. Spatial regulators for bacterial cell division self-organize into surface waves in vitro. Science 320, 789-792 (2008).

14. Wu, F., van Schie, B. G. C., Keymer, J. E. \& Dekker, C. Symmetry and scale orient Min protein patterns in shaped bacterial sculptures. Nat. Nanotechnol. 10, 719-726 (2015).

15. Vecchiarelli, A. G. et al. Membrane-bound MinDE complex acts as a toggle switch that drives Min oscillation coupled to cytoplasmic depletion of MinD. Proc. Natl Acad. Sci. USA 113, E1479-E1488 (2016).

16. Glock, P. et al. Stationary patterns in a two-protein reaction-diffusion system. ACS Synth. Biol. 8, 148-157 (2019).

17. Huang, K. C., Meir, Y. \& Wingreen, N. S. Dynamic structures in Escherichia coli: spontaneous formation of MinE rings and MinD polar zones. Proc. Natl Acad. Sci. USA 100, 12724-12728 (2003).

18. Halatek, J. \& Frey, E. Highly canalized MinD transfer and MinE sequestration explain the origin of robust MinCDE-protein dynamics. Cell Rep. 1, 741-752 (2012).

19. Denk, J. et al. MinE conformational switching confers robustness on self-organized Min protein patterns. Proc. Natl Acad. Sci. USA 115, 4553-4558 (2018)

20. Glock, P., Brauns, F., Halatek, J., Frey, E. \& Schwille, P. Design of biochemical pattern forming systems from minimal motifs. eLife 8, e48646 (2019).

21. Halatek, J. \& Frey, E. Rethinking pattern formation in reaction-diffusion systems. Nat. Phys. 14, 507-514 (2018).

22. Zieske, K. \& Schwille, P. Reconstitution of self-organizing protein gradients as spatial cues in cell-free systems. eLife 3, e03949 (2014).

23. Caspi, Y. \& Dekker, C. Mapping out Min protein patterns in fully confined fluidic chambers. eLife 5, e19271 (2016). 
24. Raskin, D. M. \& De Boer, P. A. J. MinDE-dependent pole-to-pole oscillation of division inhibitor MinC in Escherichia coli. J. Bacteriol. 181, 6419-6424 (1999).

25. Loose, M., Fischer-Friedrich, E., Herold, C., Kruse, K. \& Schwille, P. Min protein patterns emerge from rapid rebinding and membrane interaction of MinE. Nat. Struct. Mol. Biol. 18, 577-583 (2011).

26. Hale, C., Meinhardt, H. \& de Boer, P. Dynamic localisation cycle of the cell division regulator MinE in Escherichia coli. EMBO J. 20, 1563-1572 (2001).

27. Hu, Z., Mukherjee, A., Pichoff, S. \& Lutkenhaus, J. The MinC component of the division site selection system in Escherichia coli interacts with FtsZ to prevent polymerization. Proc. Natl Acad. Sci. USA 96, 14819-14824 (1999).

28. Mcquillen, R. \& Xiao, J. Insights into the structure, function and dynamics of the bacterial cytokinetic FtsZ-ring. Annu. Rev. Biophys. 49, 311-343 (2020)

29. Khmelinskaia, A., Franquelim, H. G., Petrov, E. P. \& Schwille, P. Effect of anchor positioning on binding and diffusion of elongated 3D DNA nanostructures on lipid membranes. J. Phys. D 49, 194001 (2016).

30. Marenduzzo, D., Finan, K. \& Cook, P. R. The depletion attraction: an underappreciated force driving cellular organization. J. Cell Biol. 175, 681-686 (2006)

31. Asakura, S. \& Oosawa, F. On interaction between two bodies immersed in a solution of macromolecules. J. Chem. Phys. 22, 1255-1256 (1954).

32. Miyagi, A., Ramm, B., Schwille, P. \& Scheuring, S. High-speed AFM reveals the inner workings of the MinDE protein oscillator. Nano Lett. 18, 288-296 (2018)

33. Scheuring, S., Müller, D. J., Ringler, P., Heymann, J. B. \& Engel, A. Imaging streptavidin 2D crystals on biotinylated lipid monolayers at high resolution with the atomic force microscope. J. Microsc. 193, 28-35 (1999).

34. Balian, R. From Microphysics to Macrophysics (Springer, 2007).

35. Maxwell, J. C. IV On the dynamical theory of gases. Phil. Trans. R. Soc. Lond. 157, 49-88 (1866)

36. Stefan, J. Über das Gleichgewicht und die Bewegung, insbesondere die Diffusion von Gasgemengen. Sitzber. Akad. Wiss. Wien. 63, 63-124 (1871).

37. Schweizer, J. et al. Geometry sensing by self-organized protein patterns. Proc. Natl Acad. Sci. USA 109, 15283-15288 (2012).

38. Terbush, A. D., Porzondek, C. A. \& Osteryoung, K. W. Functional analysis of the chloroplast division complex using Schizosaccharomyces pombe as a heterologous expression system. Microsc. Microanal. 22, 275-289 (2016).

39. Anderson, J. L., Lowell, M. E. \& Prieve, D. C. Motion of a particle generated by chemical gradients. Part 1. Non-electrolytes. J. Fluid Mech. 117, 107-121 (1982).

40. Prieve, D. C., Anderson, J. L. \& Lowell, M. E. Motion of a particle generated by chemical gradients. Part 2. Electrolytes. J. Fluid Mech. 148, 247-269 (1984).

41. Prieve, D. C., Malone, S. M., Khair, A. S., Stout, R. F. \& Kanj, M. Y. Diffusiophoresis of charged colloidal particles in the limit of very high salinity. Proc. Natl Acad. Sci. USA 116, 18257-18262 (2019).

42. Shin, S. et al. Size-dependent control of colloid transport via solute gradients in dead-end channels. Proc. Natl Acad. Sci. USA 113, 257-261 (2016).

43. Paustian, J. S. et al. Direct measurements of colloidal solvophoresis under imposed solvent and solute gradients. Langmuir 31, 4402-4410 (2015).
44. Illien, P., Golestanian, R. \& Sen, A. 'Fuelled' motion: phoretic motility and collective behaviour of active colloids. Chem. Soc. Rev. 46, 5508-5518 (2017).

45. Abécassis, B., Cottin-Bizonne, C., Ybert, C., Ajdari, A. \& Bocquet, L.

Boosting migration of large particles by solute contrasts. Nat. Mater. 7 , 785-789 (2008)

46. Palacci, J., Abécassis, B., Cottin-Bizonne, C., Ybert, C. \& Bocquet, L. Colloidal motility and pattern formation under rectified diffusiophoresis. Phys. Rev. Lett. 104, 138302 (2010).

47. Marbach, S. \& Bocquet, L. Osmosis, from molecular insights to large-scale applications. Chem. Soc. Rev. 48, 3102-3144 (2019).

48. Shi, N., Nery-Azevedo, R., Abdel-Fattah, A. I. \& Squires, T. M. Diffusiophoretic focusing of suspended colloids. Phys. Rev. Lett. 117, 258001 (2016).

49. Golestanian, R. Phoretic active matter. Preprint at https://arxiv.org/ pdf/1909.03747v3.pdf (2019).

50. Duncan, J. B. \& Toor, H. L. An experimental study of three component gas diffusion. AIChE J. 8, 38-41 (1962).

51. Krishna, R. \& Wesselingh, J. A. The Maxwell-Stefan approach to mass transfer. Chem. Eng. Sci. 52, 861-911 (1997).

52. Agudo-Canalejo, J., Illien, P. \& Golestanian, R. Phoresis and enhanced diffusion compete in enzyme chemotaxis. Nano Lett. 18, 2711-2717 (2018).

53. Adeleke-Larodo, T., Agudo-Canalejo, J. \& Golestanian, R. Chemical and hydrodynamic alignment of an enzyme. J. Chem. Phys. 150, 115102 (2019).

54. Banigan, E. J., Gelbart, M. A., Gitai, Z., Wingreen, N. S. \& Liu, A. J. Filament depolymerization can explain chromosome pulling during bacterial mitosis. PLoS Comput. Biol. 7, e1002145 (2011).

55. Sear, R. P. Diffusiophoresis in cells: a general nonequilibrium, nonmotor mechanism for the metabolism-dependent transport of particles in cells. Phys. Rev. Lett. 122, 128101 (2019).

56. Gerganova, V. et al. Cell patterning by secretion-induced plasma membrane flows. Preprint at bioRxiv https://doi.org/2020.12.18.423457 (2020).

57. Yang, Y. \& Wu, M. Rhythmicity and waves in the cortex of single cells. Phil. Trans. R. Soc. B 373, 20170116 (2018)

58. Hyman, A. A., Weber, C. A. \& Jülicher, F. Liquid-liquid phase separation in biology. Annu. Rev. Cell Dev. Biol. 30, 39-58 (2014).

Publisher's note Springer Nature remains neutral with regard to jurisdictional claims in published maps and institutional affiliations.

Open Access This article is licensed under a Creative Commons

Attribution 4.0 International License, which permits use, sharing, adaptation, distribution and reproduction in any medium or format, as long as you give appropriate credit to the original author(s) and the source, provide a link to the Creative Commons license, and indicate if changes were made. The images or other third party material in this article are included in the article's Creative Commons license, unless indicated otherwise in a credit line to the material. If material is not included in the article's Creative Commons license and your intended use is not permitted by statutory regulation or exceeds the permitted use, you will need to obtain permission directly from the copyright holder. To view a copy of this license, visit http://creativecommons. org/licenses/by/4.0/.

(c) The Author(s), under exclusive licence to Springer Nature Limited 2021 


\section{Methods}

Theoretical description in the presence of travelling waves. We have formulated our theory, equation (1), in terms of particle fluxes. Given these fluxes $\mathbf{j}_{\mathbf{j}}$, the spatiotemporal dynamics of each molecular species is determined by the balance equation

$$
\partial_{t} c_{i}+\nabla \cdot \mathbf{j}_{i}=r_{i}(\ldots),
$$

where $r_{i}$ signify reaction terms. We considered two species, passive cargo molecules $c_{\mathrm{g}}$ that do not engage in reactions, $r_{\mathrm{g}}=0$, and active MinD proteins $c_{\mathrm{p}}$. Instead of considering specific reaction terms, we assumed that the interplay between diffusive and reactive fluxes of MinDE leads to travelling waves. Then, we took these travelling MinD protein waves with wavelength $\lambda$ and speed $v$ :

$$
c_{\mathrm{p}}(x, t)=c_{\mathrm{p}}\left(2 \pi \frac{x-v t}{\lambda}\right) \equiv c_{\mathrm{p}}(z)
$$

as a given. As specific waveform, we assumed a skewed sinusoidal curve (Fig. 5b):

$$
c_{\mathrm{p}}(z)=\frac{2}{\pi \gamma} \arctan \left[\frac{\gamma \cos z}{1-\gamma \sin z}\right],
$$

where the parameter $\gamma \in[-1 \ldots 1]$ determines the 'skewness' of the waveform. In principle, we could also choose a different waveform (for example, a sinusoidal curve that is skewed in the opposite direction). Choosing a different waveform does not change the general result of a macroscopic net transport along the wavevector, but it can change the magnitude of the transport because the local gradient magnitude is affected.

Furthermore, for simplicity, we neglected membrane saturation effects (Supplementary Information), so that the chemical potential of a particle with size $a_{i}$ reduces to $\mu_{i} \approx k_{\mathrm{B}} T \ln \left(a_{i} c_{i}\right)$. This is the same assumption as in the derivation of the fitting function for the stationary patterns, equation (3), and hence has the same limitations at high particle densities. Taken together, the spatiotemporal dynamics of the passive cargo species is determined by the following transport equation:

$$
\partial_{t} c_{\mathrm{g}}=-\boldsymbol{\nabla} \cdot \mathbf{j}_{\mathrm{g}}=\boldsymbol{\nabla} \cdot\left[D_{\mathrm{g}} \frac{\left(1+c_{\mathrm{g}} / c_{X}\right) \nabla c_{\mathrm{g}}+\left(c_{\mathrm{g}} / c_{X}\right) \nabla c_{\mathrm{p}}}{1+c_{\mathrm{g}} / c_{X}+\left(D_{\mathrm{g}} / D_{\mathrm{p}}\right)\left(c_{\mathrm{p}} / c_{X}\right)}\right]
$$

where the interaction density is given by $c_{\mathrm{x}}=\xi_{\mathrm{p}} / \zeta_{\mathrm{pg}}$ and the two diffusion coefficients are given by $D_{\mathrm{g}}=k_{\mathrm{B}} T / \xi_{\mathrm{g}}$ and $D_{\mathrm{p}}=k_{\mathrm{B}} T / \xi_{\mathrm{p}}$. We used FEniCS ${ }^{59}$ to numerically solve equation (7) in a one-dimensional geometry with no-flux boundary conditions for the cargo.

Plasmids, strains and proteins. A list of all plasmids and primers is provided in Supplementary Tables 2 and 3. The plasmids pET28a-His-MinD_MinE ${ }^{13}$, pET28a-His-EGFP-MinD ${ }^{60}$, pET28a-His-MinE ${ }^{13}$ and pET28a-MinE-His ${ }^{16}$ were used for purification of His-MinD, His-EGFP-MinD, His-MinE and MinE-His, respectively, as described in detail previously ${ }^{61}$. In brief, proteins were expressed in E. coli BL21 (DE3) and then purified via Ni-NTA affinity and size-exclusion chromatography in storage buffer (50 mM HEPES/KOH pH 7.2, $150 \mathrm{mM} \mathrm{KCl,} 10 \%$ glycerol, $0.1 \mathrm{mM}$ EDTA, $0.4 \mathrm{mM}$ tris(2-carboxyethyl)phosphine (TCEP)). Proteins were snap-frozen in liquid nitrogen and stored in small aliquots until further use at $-80^{\circ} \mathrm{C}$.

Plasmids for heterologous co-expression of MinD, MinE and model peripheral membrane proteins pREP41X-sfGFP-MinD, pREP42X-MinE, pREP41X_coex sfGFP-MinD_MinE, pREP42X-mCherry-BsMTS, pREP42X-mCherry and pREP42X-mCherry-BsMTS were constructed analogously to plasmids devised by Terbush and colleagues ${ }^{38}$. pREP41X and pREP42X, and the leucine and uracil auxotroph S. pombe strain FY61 [h- ura4-D18 leu1-32], were a kind gift from S. Forsburg, University of Southern California ${ }^{62}$. All constructs based on pREP41X and pREP42X express the respective proteins under the control of the inducible $n m t 1^{*}$ promoter. For details see Supplementary Methods.

Reconstitution in S. pombe. Details of S. pombe culture and transformation are provided in the Supplementary Methods. For image acquisition, 5-ml cultures with selective medium were inoculated with the respective strains from glycerol stocks or plates and grown for $48-72 \mathrm{~h}$ at $30^{\circ} \mathrm{C}$. Cover slides were rinsed with ethanol and $\mathrm{ddH}_{2} \mathrm{O}$, and further cleaned in a plasma cleaner with oxygen as the process gas. Subsequently, Grace Bio-LABs reusable culture well gaskets (diameter of $9 \mathrm{~mm}$, GBL103240, Sigma-Aldrich) or sticky slides (\#81818, ibidi) were attached to the clean cover slides. Poly-L-lysine solution $(0.01 \%$, P4707, Sigma-Aldrich) was added to the wells and incubated for more than $30 \mathrm{~min}$. Wells were washed with the respective media once before $10-50 \mu \mathrm{l}$ of cell suspension was added for microscopy at a constant room temperature of $23^{\circ} \mathrm{C}$.

DNA origami nanostructures. The elongated DNA origami nanostructure used here was previously designed and described in ref. ${ }^{29}$. The 20 -helix bundle with hexagonal lattice is based on the M13mp18 7,429-nucleotide-long scaffold plasmid (p7429; Bayou Biolabs) and was modified using CaDNAno ${ }^{63}$. Staple oligonucleotides, 5'-Cy3B/Cy5-functionalized oligonucleotides, the
$5^{\prime}$-cholesteryl-TEG functionalized oligonucleotides (high-purity, salt-free, Eurofins MWG Operon) and 5'-biotin-TEG functionalized oligonucleotides (Sigma-Aldrich) were purchased or diluted in Milli-Q ultrapure water at a concentration of $100 \mu \mathrm{M}$. Origami structures with $1-15$ anchors were based on the previous design ${ }^{29}$, which was further modified for functionalization with 42 anchors (Supplementary Fig. 5). The assembly of the origami structure was performed in a one-pot reaction mix as described previously ${ }^{29}$. In brief, the components were mixed at a final concentration of $20 \mathrm{nM}$ p 7429 scaffold plasmid and $200 \mathrm{nM}$ staple oligonucleotides in folding buffer $(5 \mathrm{mM}$ Tris- $\mathrm{HCl}, 1 \mathrm{mM}$ EDTA, $20 \mathrm{mM} \mathrm{MgCl}_{2}, \mathrm{pH} 8.0$ ) and annealed in a thermocycler (Mastercycler) over a 41-h cooling scheme from 65 to $40^{\circ} \mathrm{C}$. Folded nanostructures were purified to remove excess staple strands by centrifugation $(14,000 \mathrm{~g}$, three cycles for $3 \mathrm{~min}$, one cycle for $5 \mathrm{~min}$ ) in Amicon Ultra $100 \mathrm{kDa}$ molecular weight cutoff filters (Merck Millipore) using reaction buffer $(25 \mathrm{mM}$ Tris- $\mathrm{HCl} \mathrm{pH} 7.5,150 \mathrm{mM} \mathrm{KCl}$, $5 \mathrm{mM} \mathrm{MgCl}_{2}$ ). The concentration of folded Cy5-labelled origami structures was estimated by fluorescence intensity measurements using a one-drop measurement unit of a Jasco FP-8500 spectrofluorometer and subsequent comparison with an intensity calibration curve obtained for free $\mathrm{Cy} 5$ dye corrected for the multiple labelling of the origami. Cy3B-labelled DNA origami concentration was measured by absorption at $260 \mathrm{~nm}$ on a NanoDrop spectrophotometer (ThermoFisher Scientific) and related to Cy5-labelled structures of known concentrations. Cy3B/Cy5-labelled DNA origami structures contained seven Cy3B/Cy5-labelled oligonucleotides attached to extended staples on the upper facet. At the lower facet, the biotin-functionalized origami contained multiple 18-nucleotide (nt) extensions that were hybridized with complementary $5^{\prime}$ biotin-TEG-functionalized oligonucleotides ( $5^{\prime}$ biotin-TEG-AACCAGACCACCCATAGC) at defined positions. DNA origami that were bound to the membrane via cholesteryl oligonucleotides contained single or multiple 18-nt extensions on the lower facet that can hybridize with the complementary $5^{\prime}$ TEG-cholesteryl-functionalized oligonucleotides ( $5^{\prime}$ chol-TEG-AACCAGACCACCCATAGC) supplied in the self-organization assay.

Preparation of SLBs. SLBs were prepared as described in detail in refs. ${ }^{13,61}$. In brief, cover slides were rinsed with $\mathrm{ddH}_{2} \mathrm{O}$ and ethanol, and a plastic chamber was glued on top. Slides were further cleaned by plasma cleaning with oxygen as the process gas (model Zepto, Diener Electronic). Chloroform-dissolved lipids (Avanti Polar Lipids) were dried by a nitrogen stream and subsequently in a desiccator before slow rehydration at a concentration of $4 \mathrm{mg} \mathrm{ml}^{-1}$ in reaction buffer ( $25 \mathrm{mM}$ Tris-HCl pH 7.5, $150 \mathrm{mM} \mathrm{KCl}, 5 \mathrm{mM} \mathrm{MgCl}_{2}$ ). Small unilamellar vesicles were generated by sonication in a bath sonicator and subsequently added to the cleaned reaction chambers at a concentration of $0.5 \mathrm{mg} \mathrm{ml}^{-1}$. After $4 \mathrm{~min}$ of incubation on a $37^{\circ} \mathrm{C}$ warm heating block, the SLB was washed 10 times with a total of $2 \mathrm{ml}$ of wash buffer $(25 \mathrm{mM}$ Tris- $\mathrm{HCl} \mathrm{pH} \mathrm{7.5,150} \mathrm{mM} \mathrm{KCl})$ to remove excess vesicles. All mentioned concentrations refer to the final volume of the reaction chamber of $200 \mu$ l. To prepare chambers for self-organization experiments with biotin-functionalized origami, the SLB was generated with a lipid composition of 69/30/1 mol\% DOPC/DOPG/biotinyl-CAP-PE or with 70/30/0.01 mol\% DOPC/DOPG/biotinyl-CAP-PE for single-particle tracking experiments and subsequently incubated with non-labelled or Alexa568-labelled streptavidin (ThermoFisher Scientific) at a final concentration of $1 \mu \mathrm{g} \mathrm{ml}^{-1}$. After incubation for 5-10 min, unbound streptavidin was removed by washing five times with a total volume of $1 \mathrm{ml}$ of reaction buffer. The buffer was adjusted to a volume of $100 \mu \mathrm{l}$ and the origami was incubated at a final concentration of $0.1 \mathrm{nM}$ for $10 \mathrm{~min}$, before the buffer was adjusted to the final volume of $200 \mu$ l. To prepare chambers for self-organization experiments with cholesteryl-bound origami, the SLB was generated with a lipid composition of 70/30 mol\% DOPC/DOPG. The buffer was adjusted to a volume of $100 \mu \mathrm{l}$ and the $5^{\prime}$ TEG-cholesteryl-functionalized oligonucleotides for binding were added at a final concentration of $10 \mathrm{nM}$. Subsequently, the origami was added at a final concentration of $0.1 \mathrm{nM}$ and incubated for $10 \mathrm{~min}$, before buffer adjustment to $200 \mu \mathrm{l}$. For experiments involving more than one type of DNA origami, DNA origami species were premixed in DNA LoBind tubes (Eppendorf) before addition to the sample chamber at a final concentration of $50 \mathrm{pM}$ for each DNA origami, keeping the overall DNA origami concentration at $0.1 \mathrm{nM}$. Note that at these experimental conditions, DNA origami does not bind non-specifically to the lipid membrane in the absence of biotin-TEG-anchors/streptavidin or cholesteryl-TEG-anchors, due to the high net negative charge of both the SLB and DNA origami ${ }^{64,65}$.

Chromium-patterned cover slides. Chromium-patterned cover slides for patterned $\mathrm{SLBS}^{66,67}$ were generated by photolithography and metal evaporation. Cover slides were first cleaned by rinsing with pure ethanol and $\mathrm{ddH}_{2} \mathrm{O}$ and subsequently by plasma cleaning with oxygen as the process gas (model Zepto, $20-60 \mathrm{~s}, 40-50 \%$ power and $0.3 \mathrm{mbar}$ ). The vapour of bis(trimethylsilyl)amine (HDMS) was deposited on the cover slide for $2 \mathrm{~min}$ as adhesion promoter. Subsequently, positive photoresist (AZ ECI 3027, MicroChemicals) was spin-coated onto the cover slide ( 40 s, 4,000 r.p.m., start/stop acceleration 2,000 r.p.m. ${ }^{-1}$ ) resulting in an $\sim 3-\mu \mathrm{m}$-thick layer of photoresist. After pre-baking $\left(90 \mathrm{~s}, 90^{\circ} \mathrm{C}\right)$ the photoresist was patterned using ultraviolet lithography $(\mu \mathrm{PG} 101$, Heidelberg Instruments) with a 10-mm write head, nominal output power 
of $35 \mathrm{~mW}$ at a wavelength of $375 \mathrm{~nm}$, before passing a $45 \%$ attenuation filter. Afterwards, the slides were post baked $\left(60 \mathrm{~s}, 110^{\circ} \mathrm{C}\right)$ before applying developer (AZ 351B, $\mathrm{NaOH}$-based, diluted 1:4 (vol/vol), MicroChemicals) for $4 \mathrm{~min}$. Finally, slides were rinsed with $\mathrm{ddH}_{2} \mathrm{O}$ and dried with a nitrogen stream. Chromium was deposited onto these slides by evaporation at $22-33 \mathrm{~mA}$ at a rate of $\sim 1 \AA \mathrm{s}^{-1}$ to a final thickness of $\sim 30 \mathrm{~nm}$. After chromium deposition, the photoresist was lifted off in acetone with sonication for $5 \mathrm{~min}$ in a sonicator bath. Afterwards, slides were rinsed with isopropanol and dried with a nitrogen stream. SLBs were formed on the chromium-patterned slides as described above.

Self-organization assay. Self-organization assays were performed essentially as described in detail in ref. ${ }^{61}$. In short, they were performed on preformed SLBs in $200 \mu \mathrm{l}$ of reaction buffer ( $25 \mathrm{mM}$ Tris- $\mathrm{HCl} \mathrm{pH} 7.5,150 \mathrm{mM} \mathrm{KCl}, 5 \mathrm{mM} \mathrm{MgCl}$ ) supplemented with $2.5 \mathrm{mM} \mathrm{Mg-ATP} \mathrm{(stock:} 100 \mathrm{mM}$ ATP, in $100 \mathrm{mM} \mathrm{MgCl}$, adjusted to $\mathrm{pH} 7.5$ ) and at a constant room temperature of $23^{\circ} \mathrm{C}$. MinD was typically used at $1 \mu \mathrm{M}(0.3 \mu \mathrm{M}$ EGFP-MinD, $0.7 \mu \mathrm{M}$ MinD) with either $1.5 \mu \mathrm{M}$ MinE-His ${ }^{16}$ to generate quasi-stationary labyrinth patterns or $5 \mu \mathrm{M}$ His-MinE ${ }^{7,13}$ to generate regular travelling surface waves. Time series showing the initial development of patterns were acquired by starting the self-organization with Mg-ATP directly before image acquisition. Tile scans used for quantification of quasi-stationary patterns or gradients induced by regular travelling waves on unconstrained and patterned SLBs were acquired 30-60 min after the start of self-organization, in areas of the chamber that had not been imaged previously.

Single-particle tracking. Single-particle tracking of DNA origami was conducted at a concentration of DNA origami and anchors that can be described as diluted, so that interaction between individual DNA origami was minimized ${ }^{68}$. DNA origami was diluted in DNA LoBind tubes (Eppendorf) and added to a chamber at a final concentration of $0.1-1 \mathrm{pM}$ (biotin-functionalized origami, SLB with 70/30/0.01 mol\% DOPC/DOPG/biotinyl-CAP-PE; cholesteryl functionalized origami, SLB with 70/30 mol\% DOPC/DOPG, 0.1 nM 5' TEG-cholesterylfunctionalized oligonucleotides). Owing to the superior brightness and photostability, single-particle tracking was exclusively performed using Cy3Blabelled DNA origami. To further reduce photobleaching and blinking as well as photopolymerization of $\mathrm{MinD}$, single-particle tracking was performed in the presence of an oxygen scavenger system $\left(3.7 \mathrm{U} \mathrm{ml}^{-1}\right.$ pyranose oxidase, $90 \mathrm{U} \mathrm{ml}^{-1}$ catalase, $0.8 \%$ glucose $)^{69}$ and trolox. Experiments were performed in the absence of any protein or in the presence of $1 \mu \mathrm{M}$ MinD (30\% EGFP-MinD) and $2.5 \mathrm{mM}$ ATP to emulate conditions in the MinD maxima in a simplified fashion. For single-particle tracking of cargo in the presence of MinDE travelling waves, $1 \mu \mathrm{M} \mathrm{MinD}(30 \%$ EGFP-MinD) and $5 \mu \mathrm{M}$ His-MinE were used and tracking was performed more than $30 \mathrm{~min}$ after starting the reaction with $2.5 \mathrm{mM}$ ATP to obtain regular waves.

Microscopy. All images, except for single-particle tracking, were taken on a Zeiss LSM780 confocal laser scanning microscope using a Zeiss C-Apochromat $\times 40 / 1.20$ water-immersion objective, $\times 20$ air objective for chromium-patterned SLBs or $\times 60 / 1.4$ differential interference contrast (DIC) oil-immersion objective for $S$. pombe cells (all Carl Zeiss). Longer time series were acquired using the built-in autofocus system. All two- or three-colour images were acquired with alternating illumination for the $488 / 633 \mathrm{~nm}$ and $561 \mathrm{~nm}$ laser lines to avoid crosstalk. EGFP-MinD or sfGFP-MinD was excited using a $488 \mathrm{~nm}$ argon laser; Cy3B-labelled origami, Alexa568-streptavidin or mCherry in S. pombe were excited using a $561 \mathrm{~nm}$ diode-pumped solid-state (DPSS) laser and Cy5-labelled origami using a $633 \mathrm{~nm} \mathrm{He}-\mathrm{Ne}$ laser. Images were typically recorded with a pinhole size of 2.6-4 Airy units for the EGFP and origami channels, 1 Airy unit for the streptavidin channel, $512 \times 512$-pixel resolution and a pixel dwell time of $1.27 \mu$ s. Time series were typically acquired with $\sim 14$-s intervals for unconstrained SLBs or 4-s intervals for chromium-patterned SLBs and S. pombe cells. For single-particle tracking of DNA, origami images were acquired on a custom-built total internal reflection fluorescence microscope $(\text { TIRFM })^{70}$ using a NIKON SR Apo TIRF $\times 100 / 1.49$ oil-immersion objective, constructed around a Nikon Ti-S microscope body (both Nikon). Two laser lines (490 nm (Cobolt Calypso, $50 \mathrm{~mW}$ nominal) and $561 \mathrm{~nm}$ (Cobolt Jive, $50 \mathrm{~mW}$ nominal), Cobolt $\mathrm{AB}$ )) were controlled in power and timing (AOTF, Gooch \& Housego TF-525-250) and spatially filtered (kineFLEX-P-3-S405.640-0.7-FCS-P0, Qioptiq). The beam was further collimated, expanded (10X) and focused on the objective's back aperture by standard achromatic doublet lenses. The TIRF angle was controlled by precise parallel offset of the excitation beam (Q545, PI). The emission light was notch-filtered to remove residual excitation light, spectrally separated by a dichroic beamsplitter (T555lpxr-UF1, Chroma Technology Cooperation), bandpass-filtered 525/50 and 593/46 (both Chroma), respectively, and repositioned on two halves of the electron-multiplying charge-coupled device (EMCCD) camera (Andor iXon Ultra 897, Andor Technologies). Images were recorded with Andor Solis software (version 4.28, Andor Technologies).

Image analysis. All images were processed using Fiji (version v1.52p), MATLAB (R2018a, The Math-Works) or Python (Python Software Foundation). Brightness or contrast adjustments of all displayed images were applied homogeneously.

For line plots, the images were smoothed with a Gaussian filter with pixel width of 2 in Fiji.
For the cross-correlation plots, the images were smoothed with a Gaussian kernel of pixel width 1 . The theoretical models were formulated as boundary-value problems and solved in a one-dimensional geometry using a finite-difference scheme using $\mathrm{SciPy}^{71}$. Curve fitting was performed with $\mathrm{lmfit}^{72}$.

Single-particle tracking analysis. Analysis of single-particle tracking was conducted as described previously using previously established code ${ }^{73}$. In brief, a custom-written MATLAB code was used to detect DNA origami fluorescence in each frame and extract its position. Origami trajectories on the membrane were analysed using jump-distance analysis ${ }^{74,75}$. The distances between particle locations between subsequent frames were analysed and diffusion coefficients of particle ensembles were obtained by fitting the cumulative histograms. As, usually, some of the origami in the field of view were immobile and did not diffuse, cumulative histograms of obtained jump distances were fitted with two components, where for the second component the upper boundary was set to $0.1 \mu \mathrm{m}^{2} \mathrm{~s}^{-1}$, and usually resulted in diffusion coefficients of less than $0.01 \mu \mathrm{m}^{2} \mathrm{~s}^{-1}$.

Analysis of MinDE-dependent transport. Analysis of fluorescence intensities and contrast was essentially performed as described earlier ${ }^{7}$. In brief, tile scans were imported into Fiji, where the EGFP-MinD channel was used for segmentation to generate a binary mask of the patterns. The original non-modified images from the two or three spectral channels were analysed based on the binary mask using a custom-written MATLAB code. The average fluorescence intensity in the Alexa568-streptavidin or origami-Cy5 and EGFP-MinD spectral channel was obtained by pooling the means of individual images from one independent experiment. All means from one independent experiment and condition were pooled together. All fluorescence intensity values from one experimental set were normalized to the fluorescence intensity values obtained for the respective origami with one anchor. The Michelson contrast of the resulting cargo patterns was calculated for every individual image as the difference between the average intensity in the MinD minima and MinD maxima divided by the sum of the average intensities in the MinD maxima and minima. The contrast of the MinD patterns was calculated for every individual image as the difference between the average intensity in the MinD maxima and MinD minima divided by the sum of the average intensities in the MinDE minima and maxima.

Analysis of fluorescence profiles on chromium-patterned SLBs. Time-series from chromium-patterned SLB patches with regular travelling MinDE waves that aligned along the longest axis of the patch were selected and averaged in Fiji. The resulting average intensity of the cargo was plotted along the wavevector of the MinDE waves (that is, the longest axis of the patch) and exported as csv files. Subsequently, using MATLAB, the fluorescence intensity profiles were aligned at the beginning based on the increase of the signal, and the average profile was generated.

Reporting Summary. Further information on research design is available in the Nature Research Reporting Summary linked to this Article.

\section{Data availability}

The raw images that support the findings of this study are available at Edmond with the identifier https://dx.doi.org/10.17617/3.5n or from the corresponding authors upon request. Source data are provided with this paper.

\section{Code availability}

The code for the analysis is available at Edmond with the identifier https://dx.doi. org/10.17617/3.5n or from the corresponding authors upon request.

\section{References}

59. Logg, A., Mardal, K. A. \& Wells, G. N. Automated Solution of Differential Equations by the Finite Element Method (Springer, 2012).

60. Zieske, K., Schweizer, J. \& Schwille, P. Surface topology assisted alignment of Min protein waves. FEBS Lett. 588, 2545-2549 (2014).

61. Ramm, B., Glock, P. \& Schwille, P. In vitro reconstitution of self-organizing protein patterns on supported lipid bilayers. J. Vis. Exp. 137, 58139 (2018).

62. Forsburg, S. L. Comparison of Schizosaccharomyces pombe expression systems. Nucleic Acids Res. 21, 2955-2956 (1993).

63. Douglas, S. M. et al. Rapid prototyping of 3D DNA-origami shapes with caDNAno. Nucleic Acids Res. 37, 5001-5006 (2009).

64. Czogalla, A. et al. Switchable domain partitioning and diffusion of DNA origami rods on membranes. Faraday Discuss. 161, 31-43 (2013).

65. Khmelinskaia, A., Mücksch, J., Petrov, E. P., Franquelim, H. G. \& Schwille, P. Control of membrane binding and diffusion of cholesteryl-modified DNA origami nanostructures by DNA spacers. Langmuir 34, 14921-14931 (2018).

66. Groves, J. T., Ulman, N. \& Boxer, S. G. Micropatterning fluid lipid bilayers on solid supports. Science 275, 651-653 (1997).

67. Groves, J. T., Ulman, N., Cremer, P. S. \& Boxer, S. G. Substrate-membrane interactions: mechanisms for imposing patterns on a fluid bilayer membrane. Langmuir 14, 3347-3350 (1998). 
68. Czogalla, A., Kauert, D. J., Seidel, R., Schwille, P. \& Petrov, E. P. DNA origami nanoneedles on freestanding lipid membranes as a tool to observe isotropicnematic transition in two dimensions. Nano Lett. 15, 649-655 (2015).

69. Swoboda, M. et al. Enzymatic oxygen scavenging for photostability without pH drop in single-molecule experiments. ACS Nano 6, 6364-6369 (2012).

70. Mücksch, J. et al. Quantifying reversible surface binding via surface-integrated fluorescence correlation spectroscopy. Nano Lett. 18, 3185-3192 (2018).

71. Virtanen, P. et al. SciPy 1.0: fundamental algorithms for scientific computing in Python. Nat. Methods 17, 261-272 (2020).

72. Newville, M. et al. lmfit/lmfit-py 1.0.0 (2019); https://doi.org/10.5281/ ZENODO.3588521

73. Weimann, L. et al. A quantitative comparison of single-dye tracking analysis tools using Monte Carlo simulations. PLoS ONE 8, e64287 (2013).

74. Schütz, G. J., Schindler, H. \& Schmidt, T. Single-molecule microscopy on model membranes reveals anomalous diffusion. Biophys. J. 73, 1073-1080 (1997).

75. Kues, T., Peters, R. \& Kubitscheck, U. Visualization and tracking of single protein molecules in the cell nucleus. Biophys. J. 80, 2954-2967 (2001).

\section{Acknowledgements}

We thank P. Altpeter (CeNS Munich) for supporting us with the fabrication of chromium-patterned slides. We thank MPIB Core Facility for assistance in protein purification and S. Forsburg (University of Southern California) for supplying S. pombe strains and plasmids. We further thank F. Brauns, H. Franquelim and J. Mücksch for helpful discussions. We acknowledge financial support by the German Research Foundation (DFG) through TRR 174 (Project ID No. 269423233) and SFB1032 (Project ID No. 201269156) and the Excellence Cluster ORIGINS under Germany's Excellence
Strategy (EXC-2094-390783311). B.R., A.K. and A.G. were supported by a DFG fellowship through the Graduate School of Quantitative Biosciences Munich (QBM) P.S. acknowledges the support of the research network MaxSynBio via a joint funding initiative of the German Federal Ministry of Education and Research (BMBF) and the Max Planck Society.

\section{Author contributions}

B.R., A.G., E.F. and P.S. conceived the study. A.G. and E.F. designed the theoretical analysis. A.G. conducted the theoretical analysis. B.R., A.K. and P.B. designed experiments. B.R. performed all experiments. A.K. designed DNA origami. A.K. and B.R. prepared origami. H.E. and B.R. fabricated chromium-patterned slides. K.A.G. developed single-particle tracking code. B.R., A.G., P.B. and K.A.G. analysed data. B.R., A.G., E.F and P.S. wrote the manuscript. All authors discussed and interpreted the results and revised the manuscript.

\section{Competing interests}

The authors declare no competing interests.

\section{Additional information}

Extended data is available for this paper at https://doi.org/10.1038/s41567-021-01213-3. Supplementary information The online version contains supplementary material available at https://doi.org/10.1038/s41567-021-01213-3.

Correspondence and requests for materials should be addressed to E.F. or P.S. Peer review information Nature Physics thanks Jodi Camberg and the other, anonymous, reviewer(s) for their contribution to the peer review of this work.

Reprints and permissions information is available at www.nature.com/reprints. 
a
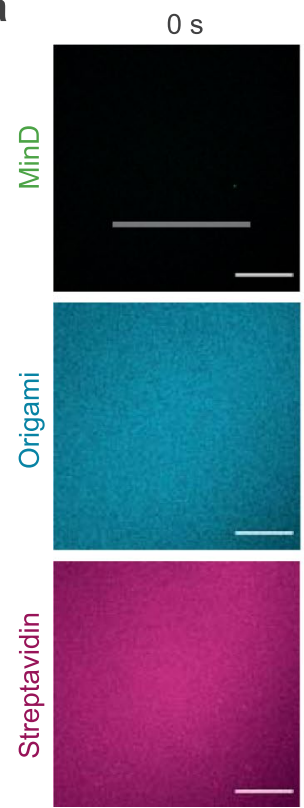

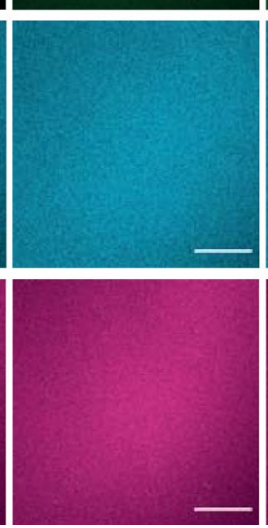

$194 \mathrm{~s}$

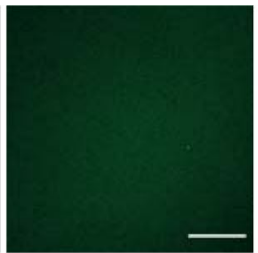

$608 s$

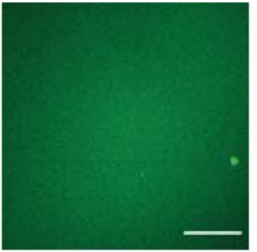

b
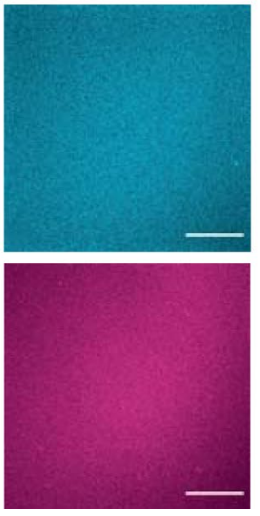

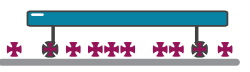

Cargo-2

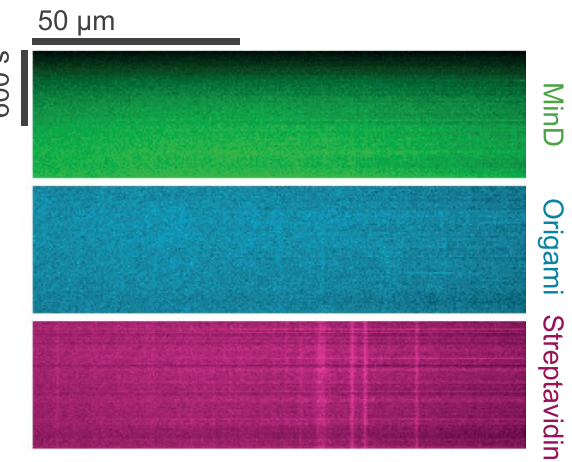

Extended Data Fig. 1 | Pattern formation of cargo depends on active self-organization by MinDE. a, Representative time series of MinD membrane binding on SLBs in the presence of cargo-2 and ATP, but in the absence of MinE ( $1 \mu \mathrm{M}$ MinD (30\% EGFP-MinD), $0.1 \mathrm{nM}$ origami-Cy5 with 2 biotinylated oligonucleotides, Alexa568-streptavidin, ATP). Scale bars, $50 \mu \mathrm{m}$. b, Kymographs of the line selection indicated in a. 
a No origami $\frac{\text { Cargo-1 }}{\text { Cargo-2 }} \underset{\text { Cargo-5 }}{\text { Cargo-15 }} \frac{\text { Cargo-28 }}{\text { Cargo-42 }}$
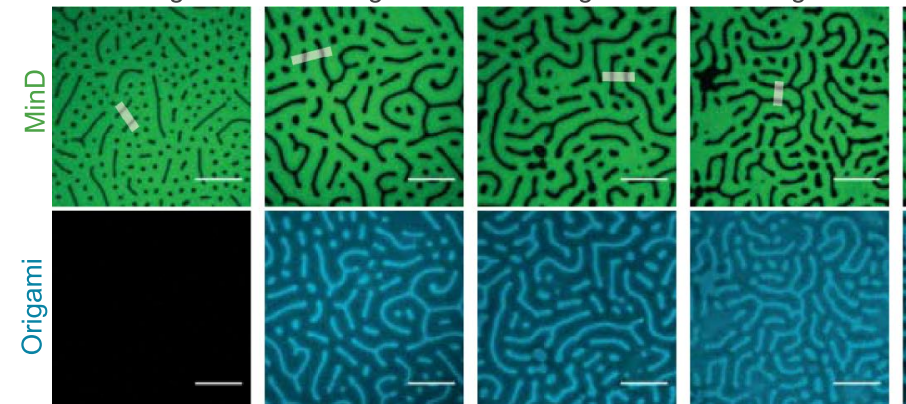

Cargo-15

Cargo-28 Cargo-42
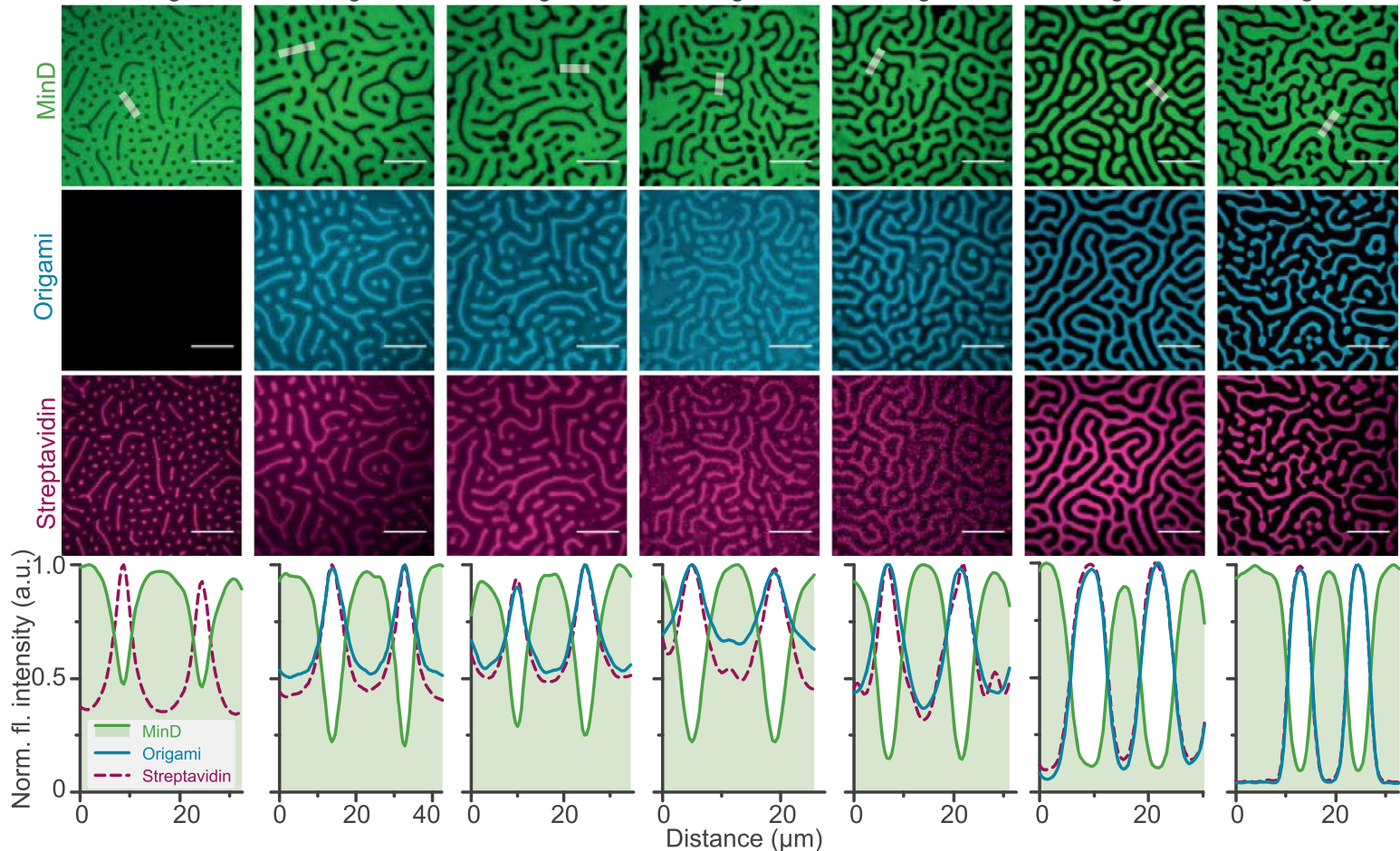

b
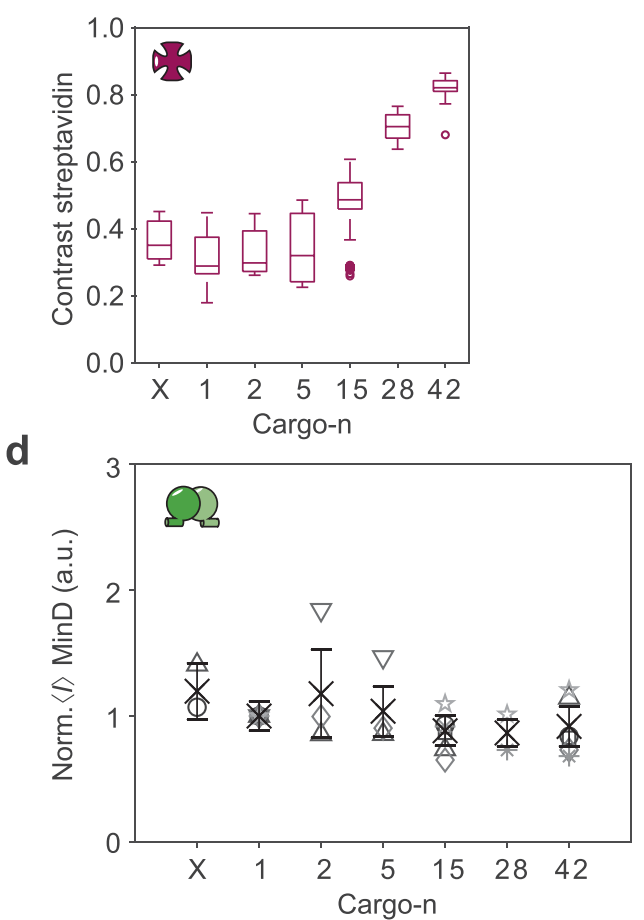

C

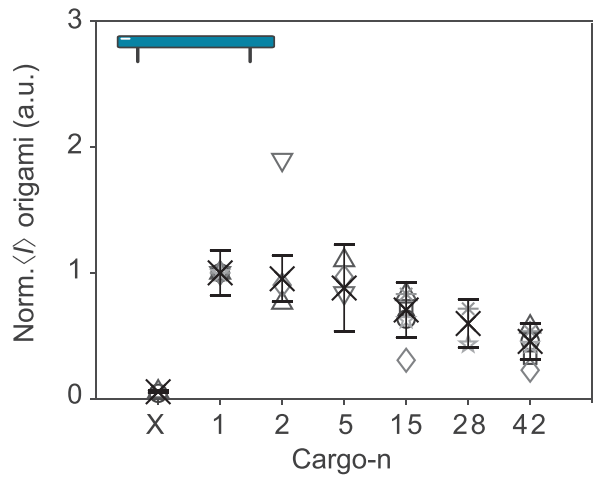

e

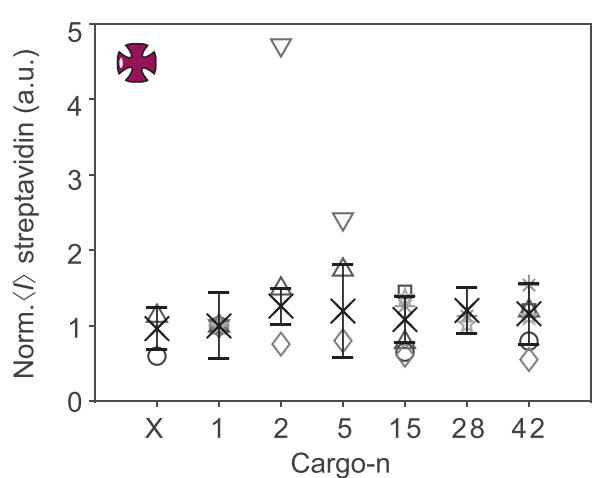

$X$ mean $\circ \exp 1$

$\square \exp 2$

$\triangle \exp 3$

$\nabla \exp 4$

$\diamond \operatorname{exp5}$

* exp6

放 $\exp 7$

Extended Data Fig. 2 | See next page for caption. 
Extended Data Fig. 2 | Extent of the MinDE-driven cargo demixing depends on the effective size (that is, membrane footprint) of the cargo. a,

Representative images and fluorescence intensity line plots (smoothed) of established MinDE labyrinth patterns and anti-correlated DNA origami and streptavidin patterns when no origami or cargo-1, cargo-2, .., cargo-42 is present ( $1 \mu \mathrm{M} \mathrm{MinD} \mathrm{(30 \%} \mathrm{EGFP-MinD),} 1.5 \mu \mathrm{M} \mathrm{MinE-His,} \mathrm{with} \mathrm{or} \mathrm{without} \mathrm{0.1} \mathrm{nM}$ origami-Cy5 with $n$ biotinylated oligonucleotides, Alexa568-streptavidin). Panels "no origami, cargo-2, cargo-15 and cargo-42" are identical to Fig. 1d. Scale bars, $50 \mu \mathrm{m}$. b. Contrast of the resulting streptavidin patterns increases with increasing number of streptavidin incorporated into the cargo. Box plot lines are median, box limits are quartiles 1 and 3 , whiskers are $1.5 \times$ interquartile range (IQR) and points are outliers. Mean fluorescence intensity of $\mathbf{c}$, DNA origami, d, EGFP-MinD and e, Alexa568-streptavidin of patterns formed when no origami or cargo-1, cargo-2, ..., cargo-42 is present; data is taken from the full image and normalised to the intensity of experiments containing cargo-1. Cross and error bars represent the mean value and standard deviation of two or more independent experiments with total number of analysed images per condition $\mathrm{N}($ No origami) $=32, \mathrm{~N}(\mathrm{Cargo}-1)=96, \mathrm{~N}(\mathrm{Cargo}-2)=41$, $\mathrm{N}($ Cargo-5) $=32, \mathrm{~N}($ Cargo-15) $=94, \mathrm{~N}($ Cargo-28) $=32, \mathrm{~N}($ Cargo -42$)=87$. 

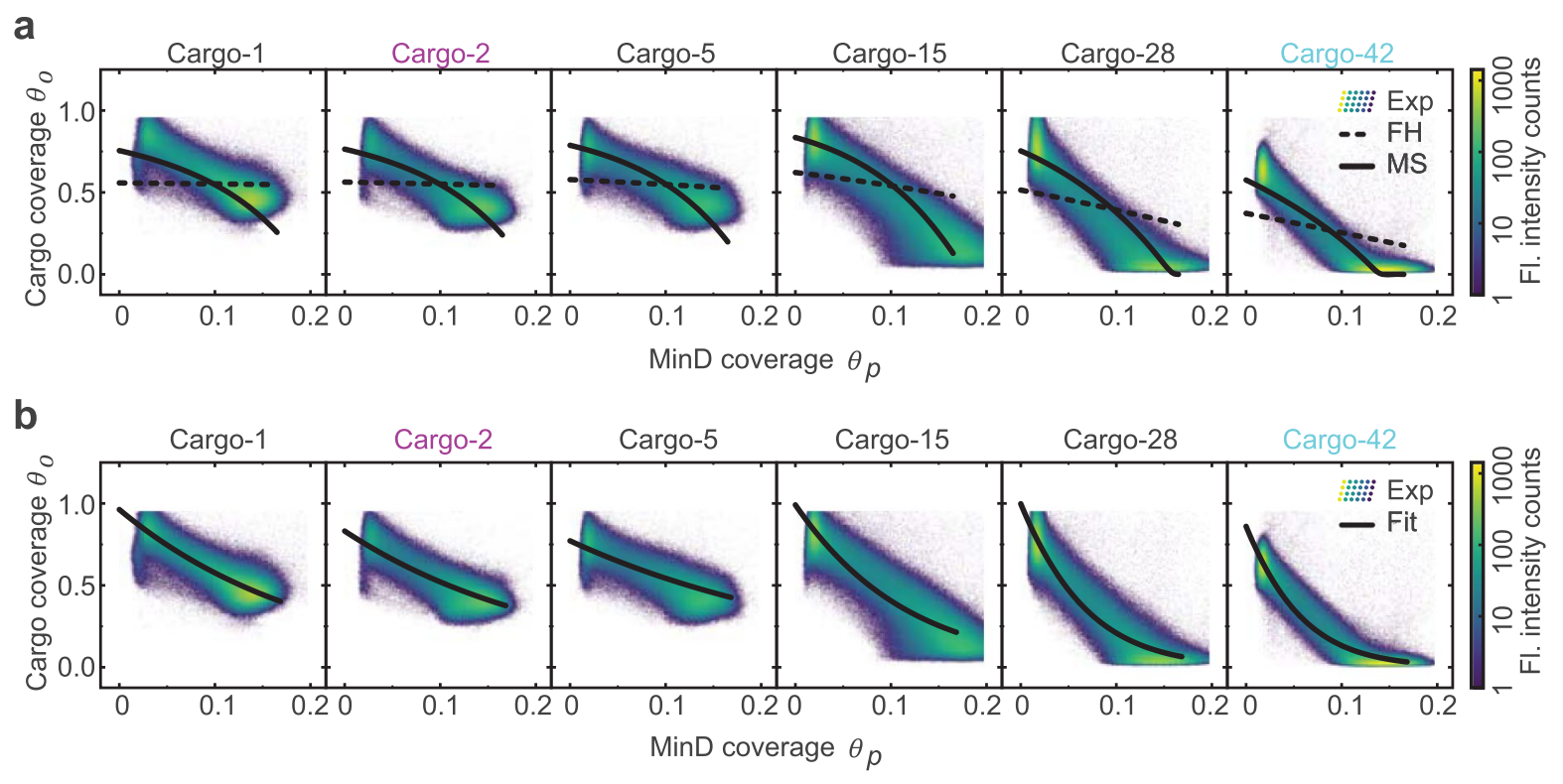

Extended Data Fig. 3 | Comparison between theory and experiment for different cargo molecules. a, Cross-correlation function between MinD coverage $\left(\theta_{p}\right)$ and DNA origami coverage $\left(\theta_{0}\right)$, for different cargo species. The color-coded 2D-histogram represents our experimental data of selected days (Exp), where the images were smoothed by a one-pixel-wide gaussian kernel. Solid and dashed lines correspond to two candidate models. The Flory-Huggins type model (FH), whose parameters are fully determined by our experiments, fails to account for cargo transport: the weak entropic sorting of streptavidin in an external gradient of proteins is not sufficient to overcome the strong repulsion of the bulky DNA origami scaffolds. Instead, we find that the phenomenological MS-type model, with an estimated interaction parameter, explains cargo transport. Model parameters: (cargo-n) average coverages $\overline{\theta_{p}}=0.0825, \overline{\theta_{s}}=\max (0.165-0.55 n / 70,0)$ and $\overline{\theta_{0}}=\min (11.55 / \mathrm{n}, 0.55)$; interaction parameter (in terms of MinD coverage) $\theta_{\times}^{-1}=200+10 n$. Surface coverages $\boldsymbol{\theta}=a c$ and surface densities $c$ are related via the particle size $a$. $\mathbf{b}$, The color-coded 2D-Histogram represents our experimental data of selected days (Exp), while the solid line (Fit) is a fit curve of our reduced model (Supplementary Information section I.5 "Analytic solution and fitting of reduced model"). 
a
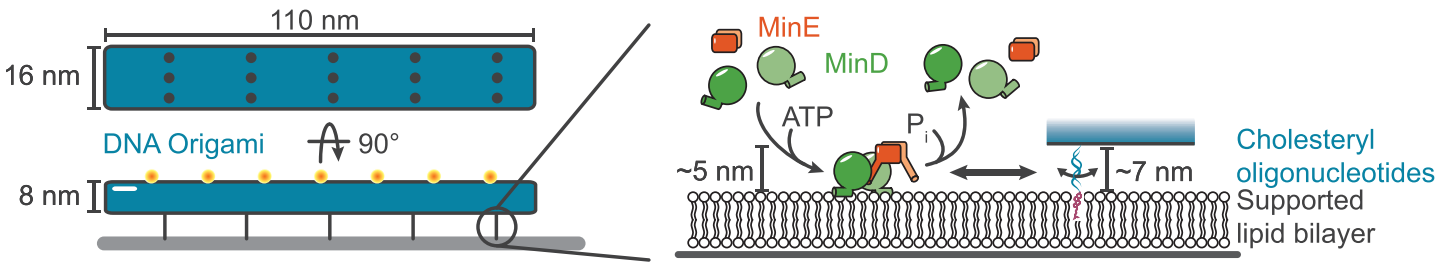

b

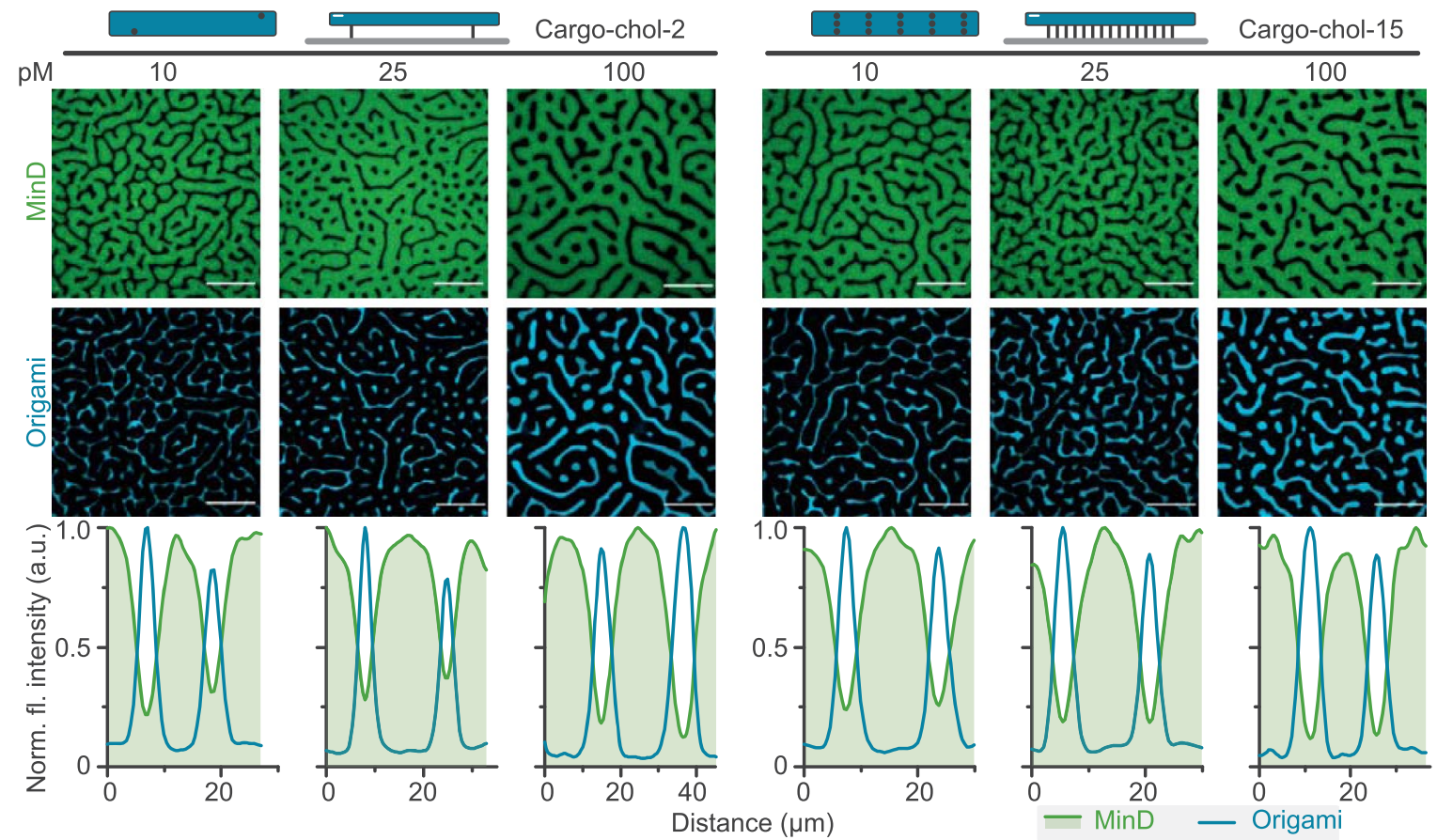

Extended Data Fig. 4 | MinDE induces pattern formation of cargo that is bound to the membrane via cholesteryl oligonucleotides. a, Schematic of the synthetic cargo consisting of a DNA origami scaffold that is bound to the membrane via cholesteryl moieties. DNA origami are bound via hybridization of DNA oligonucleotides at the indicated positions with the complementary TEG-cholesteryl oligonucleotides in the lipid membrane. $\mathbf{b}$, Representative images and fluorescence intensity line plots (smoothed) of established MinDE labyrinth patterns and anti-correlated DNA origami patterns when 10, 25 or $100 \mathrm{pM}$ of cargo-chol 2 or cargo-chol-15 are present ( $1 \mu \mathrm{M}$ MinD (30\% EGFP-MinD), $1.5 \mu \mathrm{M}$ MinE-His with 10, 25 or 100 pM origami-Cy3b with 2 or 15 hybridizing oligonucleotides, $10 \mathrm{nM}$ TEG-cholesteryl oligonucleotide). Scale bars, $50 \mu \mathrm{m}$. 
a
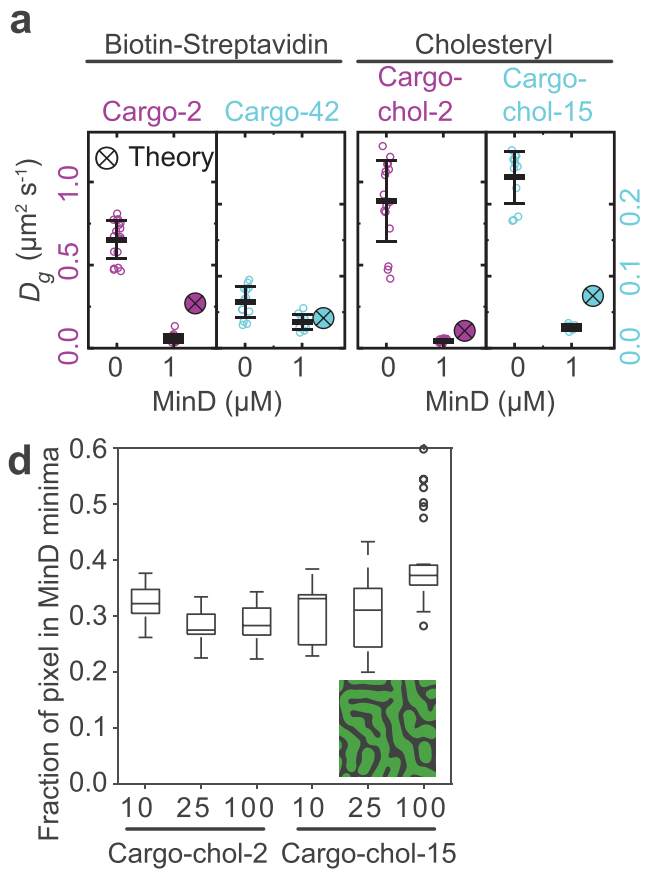

g

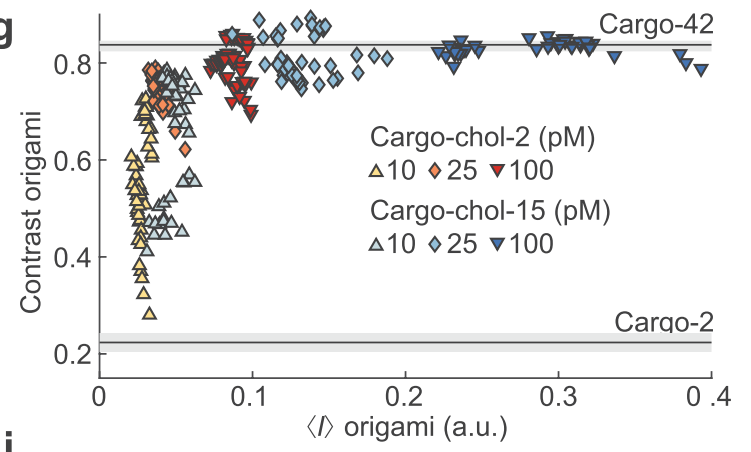

i

MinD Cargo-chol-2 Cy3B Cargo-chol-15 Cy5

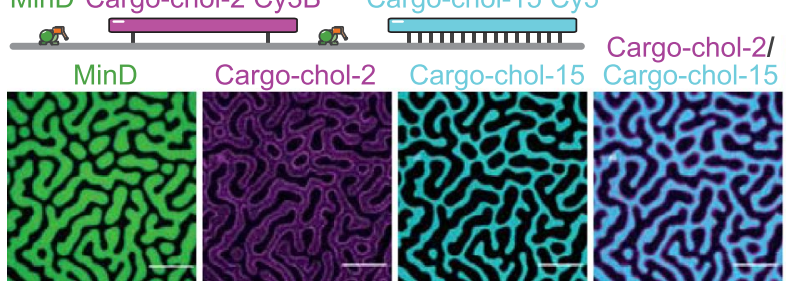

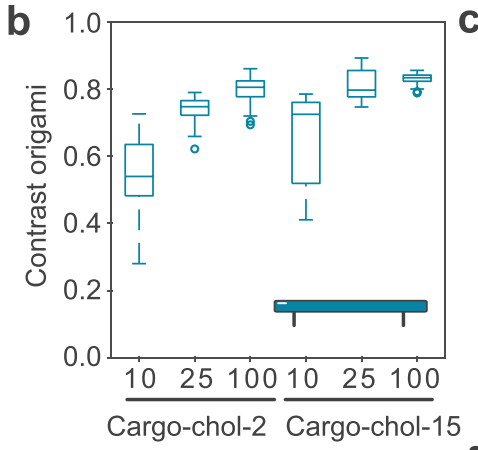

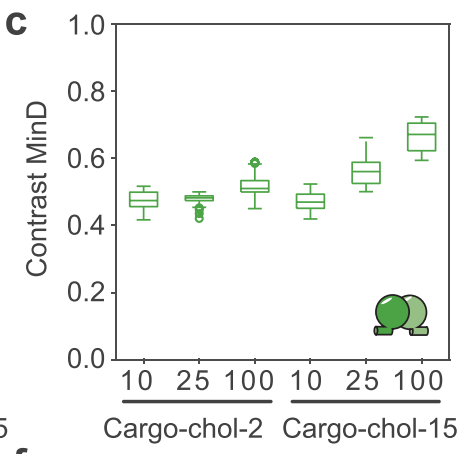

f
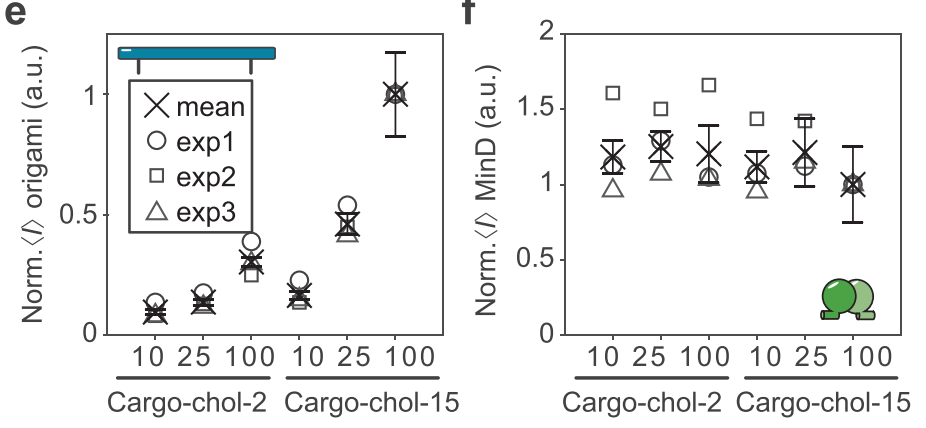

h

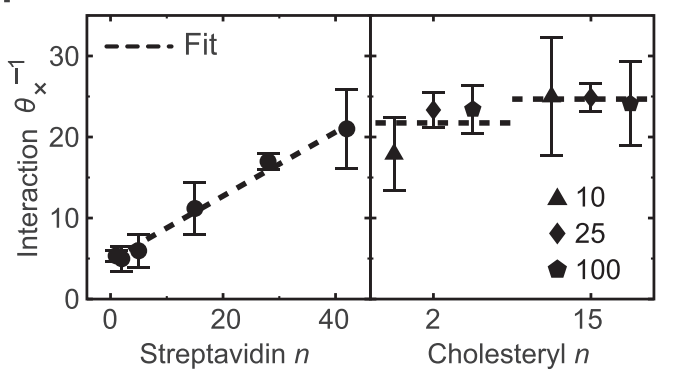

MinD/Cargo-chol-2I Cargo-chol-15

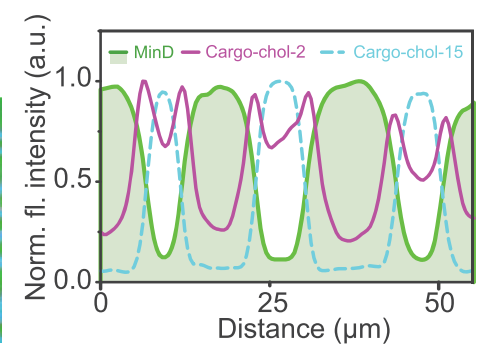

Extended Data Fig. 5 | See next page for caption. 
Extended Data Fig. 5 | MinDE-driven cargo demixing indeed depends on the effective size (membrane footprint) of the cargo and not on the diffusion coefficient. a, Cargo molecule diffusion coefficient in absence or presence of $1 \mu \mathrm{M} \mathrm{MinD}$ (with or without $1 \mu \mathrm{M}$ MinD (30\% EGFP-MinD), 0.1-1 pM origami-Cy3b with 2 or 15 hybridizing oligonucleotides, $0.1 \mathrm{nM}$ TEG-cholesteryl oligonucleotide), as obtained from single particle tracking and predicted with the fitted interaction parameters obtained in $\mathrm{h}$. Points are mean values of individual measurements $(\mathrm{M})$, line and error bars represent the mean value and standard deviation. Left part identical to Fig. 3c. Data obtained from number of sample chambers $\mathrm{S}(\mathrm{Cargo}-\mathrm{chol}-2)=7, \mathrm{~S}(\mathrm{Cargo}-\mathrm{chol}-15)=5$, $\mathrm{S}($ Cargo-chol-2, MinD)=3, S(Cargo-chol-15, MinD) =2; number of measurements $\mathrm{M}($ Cargo-chol-2)=16, M(Cargo-chol-15) $=12, \mathrm{M}($ Cargo-chol-2, $\mathrm{MinD})=14, \mathrm{M}($ Cargo-chol-15, MinD)=6; number of analysed single particle tracks N(Cargo-chol-2)=19,647, N(Cargo-chol-15) =23,365, N(Cargo-chol-2, $\mathrm{MinD})=1,879, \mathrm{~N}($ Cargo-chol-15, MinD)=8,338; average track-length TL(Cargo-chol-2)=303, TL(Cargo-chol-15) =353, TL(Cargo-chol-2, MinD)=964, $\mathrm{TL}($ Cargo-chol-15, MinD)=749; fraction of mobile DNA origami MF(Cargo-chol-2)=0.85, MF(Cargo-chol-15)=0.87, MF(Cargo-chol-2, MinD)=0.73, $\operatorname{MF}($ Cargo-chol-15, MinD)=0.67. Box plot of the contrast of $\mathbf{b}$, cargo, $\mathbf{c}$, MinD patterns, and $\mathbf{d}$, of the fraction of pixels classified as MinD minima, when 10,25 or 100 pM of cargo-chol2 or cargo-chol-15 are present ( $1 \mu \mathrm{M}$ MinD (30\% EGFP-MinD), $1.5 \mu$ M MinE-His with 10, 25 or 100 pM origami-Cy3b with 2 or 15 hybridizing oligonucleotides, $10 \mathrm{nM}$ TEG-cholesteryl oligonucleotide). Box plot lines are median, box limits are quartiles 1 and 3, whiskers are $1.5 \times$ interquartile range (IQR) and points are outliers. Mean fluorescence intensity of $\mathbf{e}$, DNA origami and $\mathbf{f}$, EGFP-MinD of the full image, normalised to the intensity of experiments with $100 \mathrm{pM}$ cargo-chol-15. Cross and error bars represent the mean value and standard deviation. $\mathbf{g}$, Increased membrane density leads to an apparent increase in contrast. Data from three independent experiments with total number of analysed images per condition $\mathrm{N}($ Cargo-chol-2, $10 \mathrm{pM})=48, \mathrm{~N}($ Cargo-chol $-2,25 \mathrm{pM})=48, \mathrm{~N}($ Cargo-chol-2, $100 \mathrm{pM})=64, \mathrm{~N}($ Cargo-chol-15, $10 \mathrm{pM})=45, \mathrm{~N}($ Cargo-chol-15, $25 \mathrm{pM})=48$, $\mathrm{N}($ Cargo-chol-15, $100 \mathrm{pM})=41$. Lines represent median and quartiles 1 and 3 of cargo-2 and cargo-42 contrast for comparison. $\mathbf{h}$, Interaction parameter (in terms of MinD coverage; surface coverages $\boldsymbol{\theta}=a c$ and surface densities $c$ are related via the particle size $a$ ) as obtained from our fitting procedure. The interaction for cargo-chol-2 and cargo-chol-15 is larger than for cargo with streptavidin building blocks. Left part is identical to Fig. 3b. The dashed line indicates a linear fit of the interaction parameter $\theta_{\times}^{-1}$ as a function of $n$. $\mathbf{i}$, Representative images of individual and overlaid channels, and line plot of indicated selection of MinDE-induced sorting of cargo-chol-2 and cargo-chol-15 (1 $\mu \mathrm{M} \mathrm{MinD} \mathrm{(30 \%} \mathrm{EGFP-MinD),} 1.5 \mu \mathrm{M}$ MinE-His with 50 pM origami-Cy3b with 2 and 50 pM origami-Cy5 with 15 hybridizing oligonucleotides, $10 \mathrm{nM}$ TEG-cholesteryl oligonucleotide). Scale bars, $50 \mu \mathrm{m}$. 
a

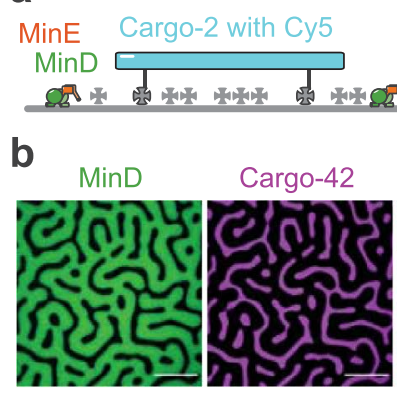

Cargo-42 with Cy3B

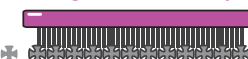

Cargo-2

Cargo-42I

MinD/

Cargo-42I Cargo-2 Cargo-2

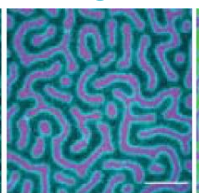

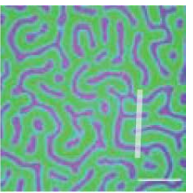

C

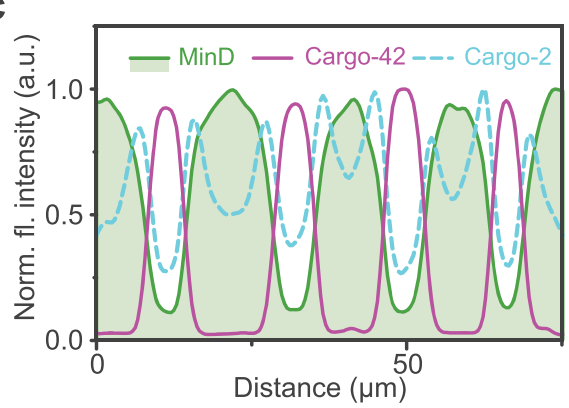

f

d
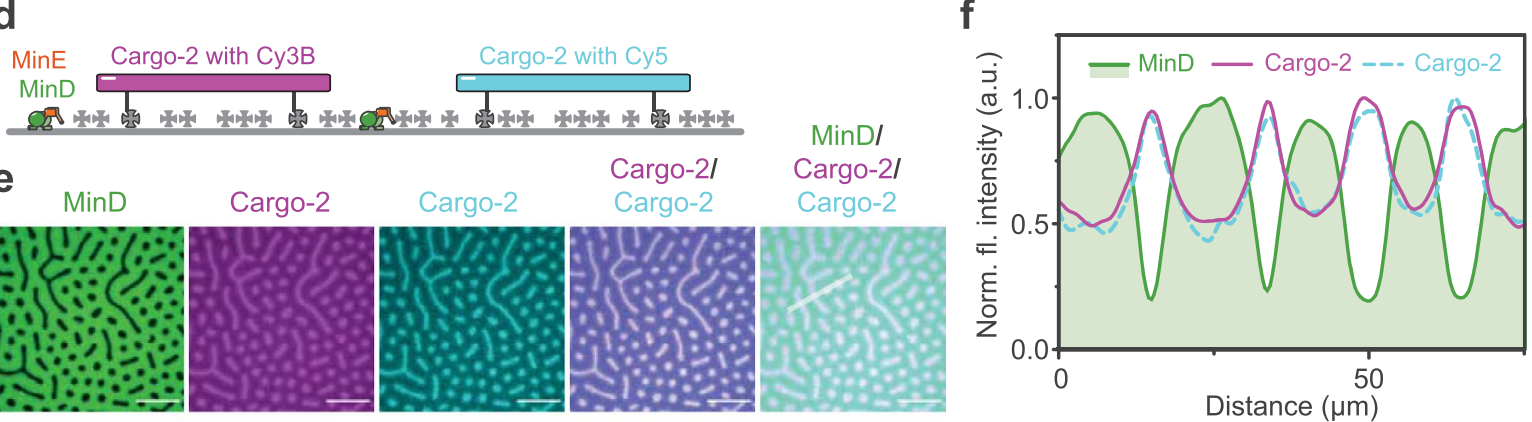

i

g

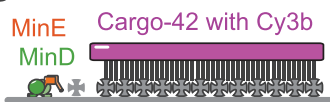

$$
\text { h }
$$
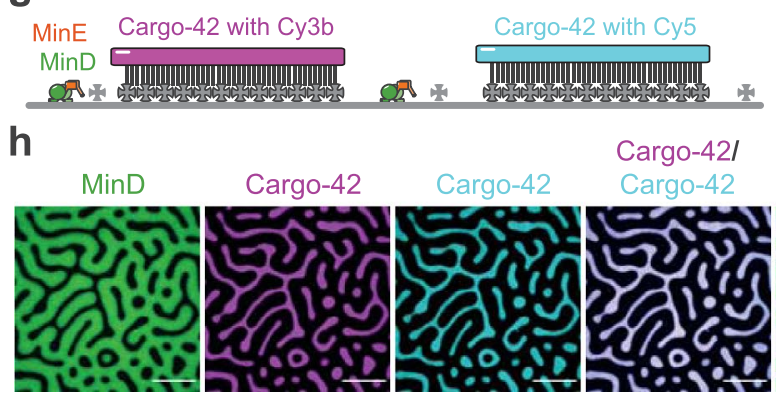

Cargo-42I
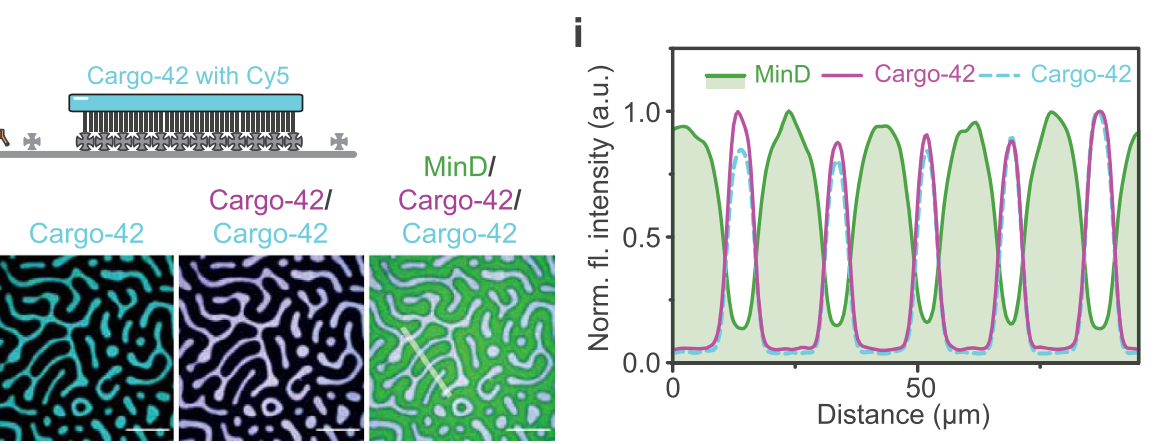

Extended Data Fig. 6 | The spatial sorting of cargo by MinDE according to the effective cargo size is not an artefact. a-c, Spatial sorting of cargo-2 and cargo- 42 by MinDE also occurs when dyes are swapped. $\mathbf{a}$, Schematic of the experimental setup. MinDE self-organization was performed in presence of two different cargo species with distinct fluorescent labels, cargo- 2 with Cy 5 and cargo- 42 with Cy3B. b, Representative images and $\mathbf{c}$, line plots of MinDE-induced sorting of cargo-2 and cargo-42 (1 $\mu \mathrm{M} \mathrm{MinD,} 1.5 \mu \mathrm{M}$ MinE-His, 50 pM origami-Cy 5 with 2 biotinylated oligonucleotides, 50 pM origami-Cy3B with 42 biotinylated oligonucleotides, non-labelled streptavidin). Experiment was performed three times under identical conditions. $\mathbf{d}$-i, MinDE-induced distributions of differentially labelled, but otherwise identical cargo are superimposable. $\mathbf{d}$, and $\mathbf{g}$, Schematic of the experimental setup: two identical cargoes are labelled with distinct dyes. Pattern formation is induced by addition of MinDE $(1 \mu \mathrm{M} \mathrm{MinD,} 1.5 \mu \mathrm{M}$ MinE-His, 50 pM origami-Cy5 and $50 \mathrm{pM}$ origami-Cy3B with either both 2 or both 42 biotinylated oligonucleotides, non-labelled streptavidin). Representative images and line plot of pattern formation in presence of $\mathbf{e}, \mathbf{f}$, two differently labelled cargo- 2 and $\mathbf{h}, \mathbf{i}$, two differently labelled cargo-42. Experiments were performed two times under identical conditions. Scale bars, $50 \mu \mathrm{m}$. 
a

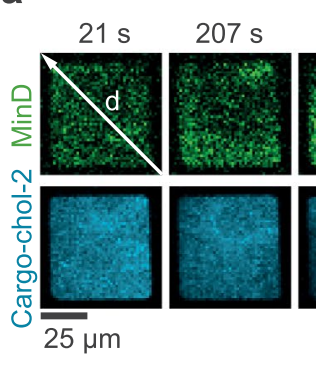

C

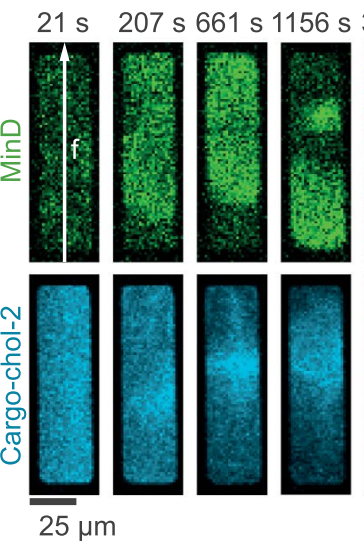

g

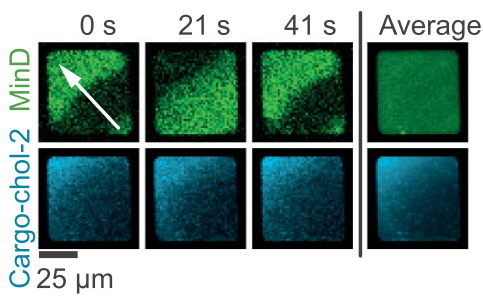

$661 \mathrm{~s}$
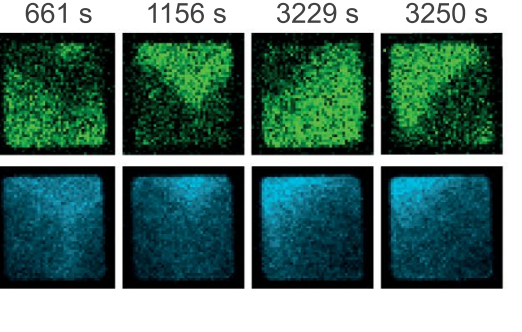

d

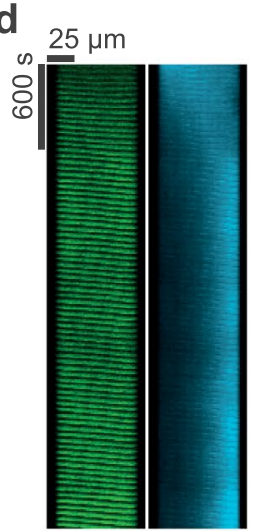

b

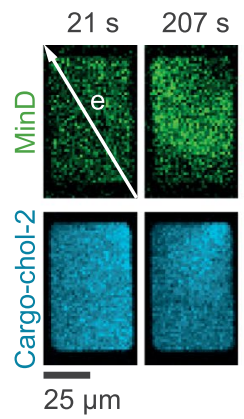

e

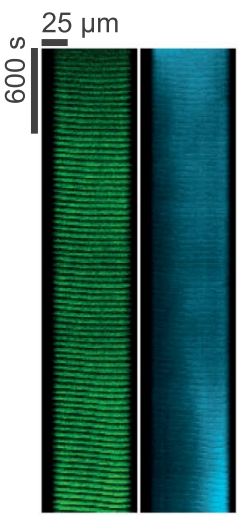

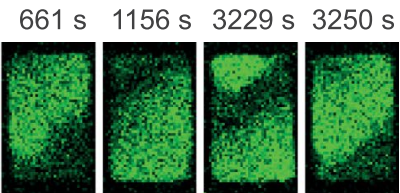

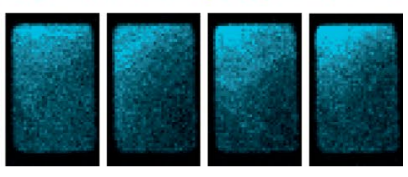

f

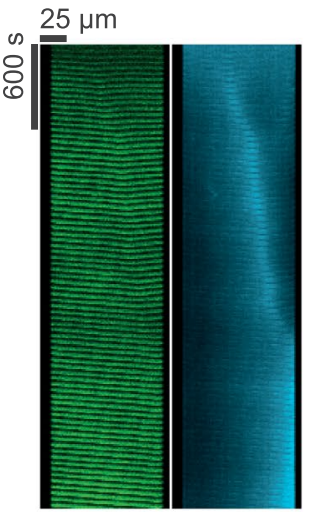

h

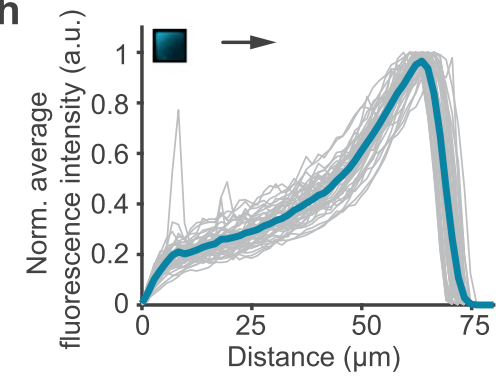

i

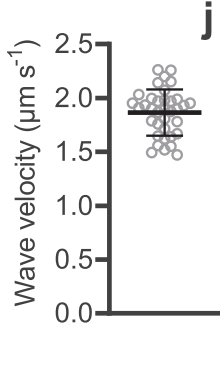

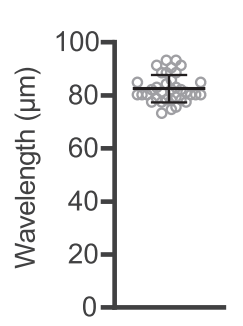

k

I
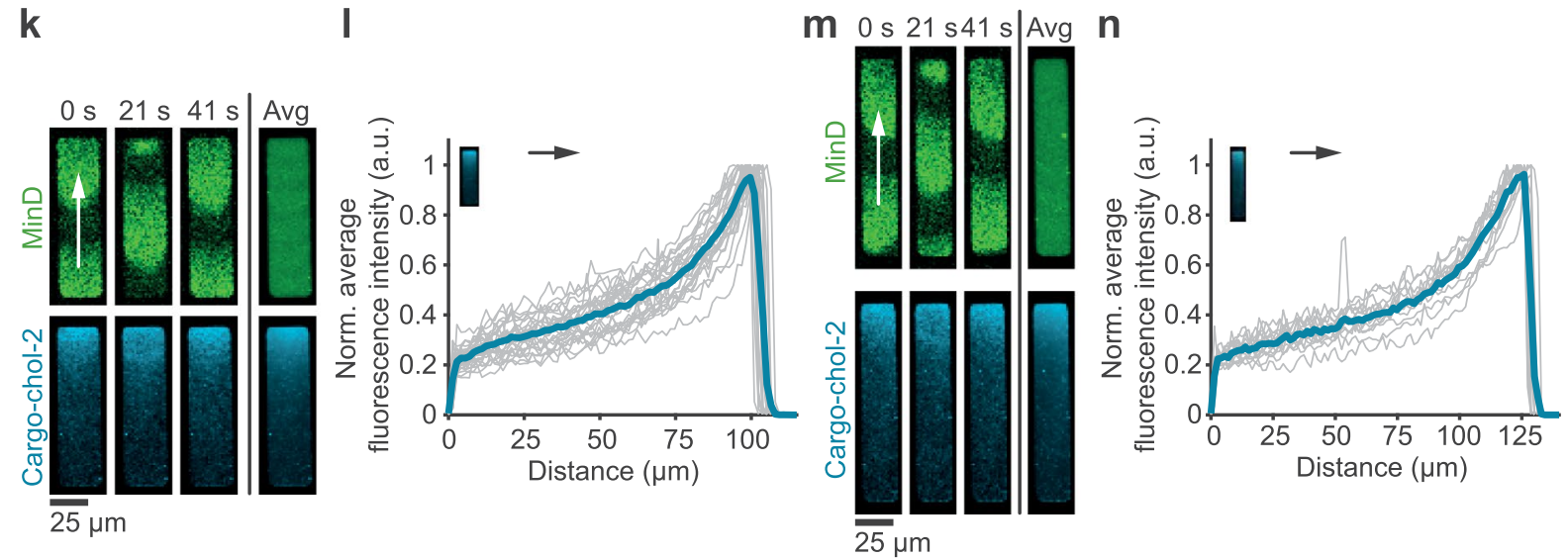

n

Extended Data Fig. 7 | Directed cargo transport by MinDE on chromium patterned bilayers. a,b,c, Representative time series and d,e,f, corresponding kymograph of MinDE traveling surface waves establishing cargo-chol-2 gradients from an initially homogeneous distribution on chromium patterned SLBs ( $1 \mu \mathrm{M}$ MinD, $5 \mu \mathrm{M}$ His-MinE, $0.1 \mathrm{nM}$ origami-Cy3b with 2 hybridizing oligonucleotides, $10 \mathrm{nM}$ TEG-cholesteryl oligonucleotide). g,k, $\mathbf{m}$, Representative time-series and average of MinDE traveling surface waves and cargo-chol-2 gradient along the wave vector after regular waves have formed ( $>30$ minutes of incubation), along the longest axis on chromium-patterned bilayers for three geometries and $\mathbf{h}, \mathbf{l}, \mathbf{n}$, the respective normalised average fluorescence intensity profiles of cargo along the wave vector in $g, k, m$. Bold, colored line represents the mean profile, generated from $N=44, N=27$ and $N=13$ membrane patches, respectively. $\mathbf{i}$, Wave velocity and $\mathbf{j}$, wavelength of MinDE waves on chromium patterned bilayers in presence of cargo-chol-2 or cargo-chol-15 with $\mathrm{N}=52$ analysed membrane patches. Arrows indicate wave direction. Scale bars, $25 \mu \mathrm{m}$. 
a

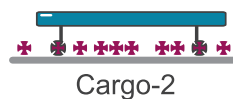

$\operatorname{Min} D$ $50 \mu \mathrm{m}$

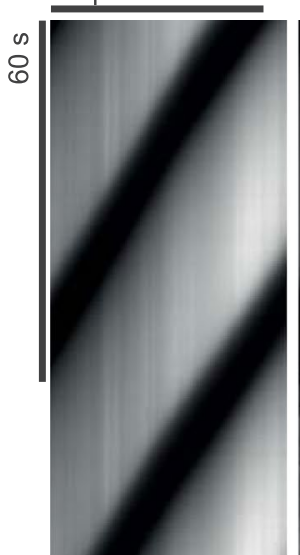

Cargo

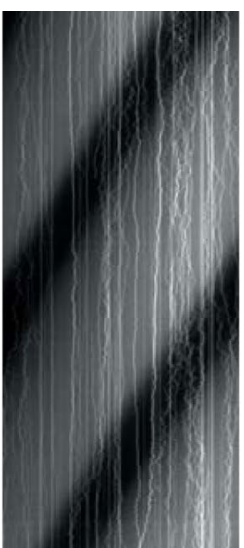

b

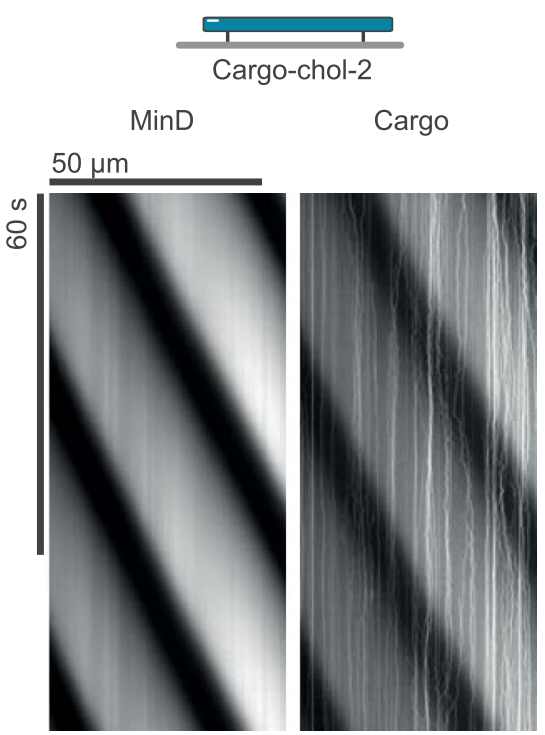

Extended Data Fig. 8 | No directed cargo movement can be observed on the single particle level. Representative kymographs of MinD waves and a, single cargo-2 and $\mathbf{b}$, single cargo-chol- 2 molecules in presence of regular directional MinDE travelling waves $(1 \mu \mathrm{M}$ MinD $(30 \%$ EGFP-MinD), $5 \mu \mathrm{M}$ His-MinE, 0.1-1 pM origami-Cy3b with 2 hybridizing oligonucleotides and $0.1 \mathrm{nM}$ TEG-cholesteryl oligonucleotide or with 2 biotinylated oligonucleotides, non-labelled streptavidin, SLB: DOPC/DOPG/Biotinyl-CAP-PE 70/30/0.01 mol\%.). 
a

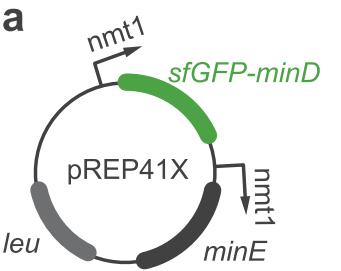

leu

sfGFP-MinD/ MinE

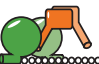

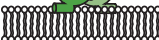

b
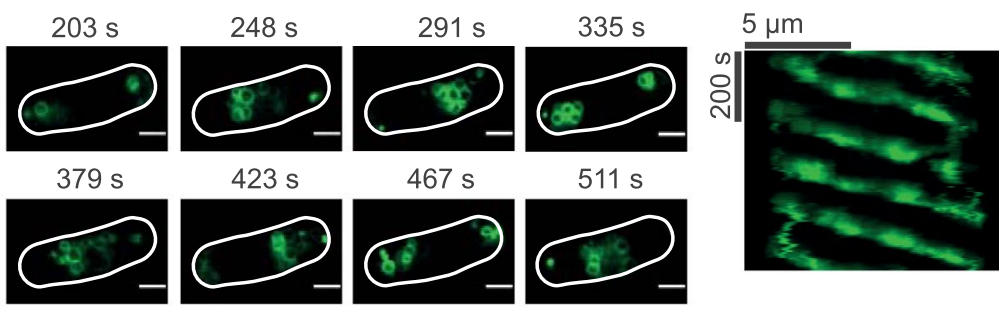

C

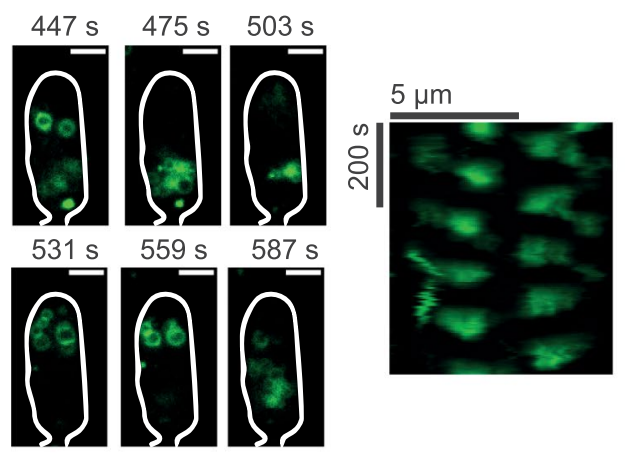

d

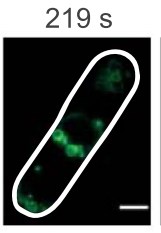

$263 \mathrm{~s}$

$307 \mathrm{~s}$

$351 \mathrm{~s}$

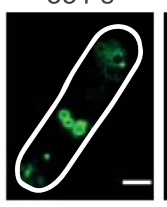

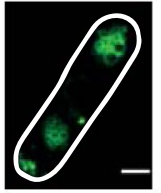

$395 \mathrm{~s}$

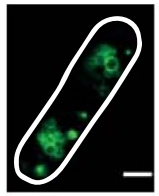

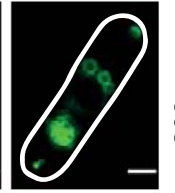

$439 \mathrm{~s}$

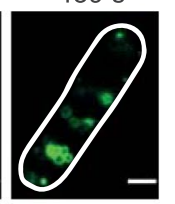

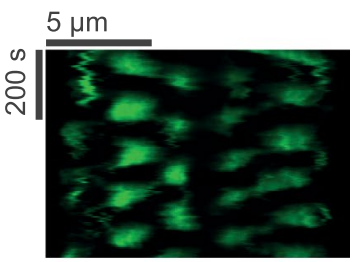

g

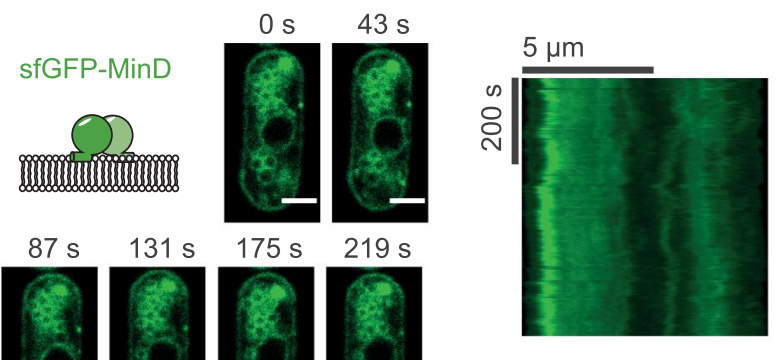

e

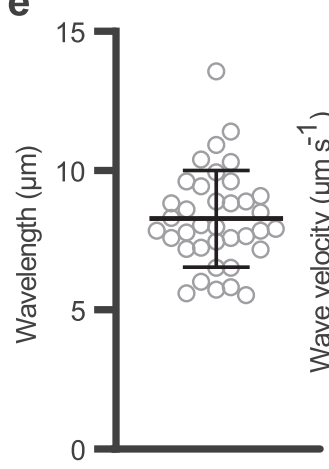

f

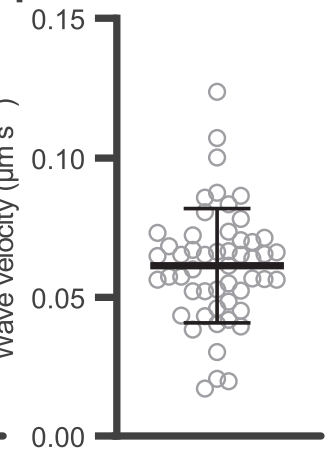

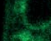

(8)

Extended Data Fig. 9 | MinDE self-organize in the fission yeast S. pombe at a similar spatiotemporal scale as in E. coli. a, Schematic of the plasmid employed for co-expression of sfGFP-MinD and MinE. b-d, Representative time-series and kymograph of S. pombe cells harbouring the co-expression plasmid for sfGFP-MinD and MinE, displaying MinD dynamics: b, travelling waves, $\mathbf{c}$, pole-to-pole like oscillations and $\mathbf{d}$, more complex dynamics. Analysis of $\mathbf{e}$, the wavelength and $\mathbf{f}$, wave velocity obtained from manually fitting the kymographs of cells displaying MinDE travelling waves. Lines represent the median and standard deviation. Data from three experiments with in total analysed cells e, $N=37$ and f, $N=52$. White boundaries represent cell outline obtained from average fluorescence images. $\mathbf{g}$, No MinD dynamics can be observed when sfGFP-MinD is expressed in S. pombe in the absence of MinE. Scale bars, $2 \mu \mathrm{m}$. 
a

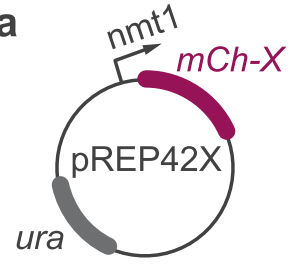

c

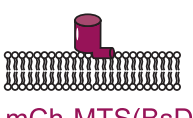

mCh-MTS(BsD)

d

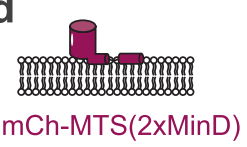

b

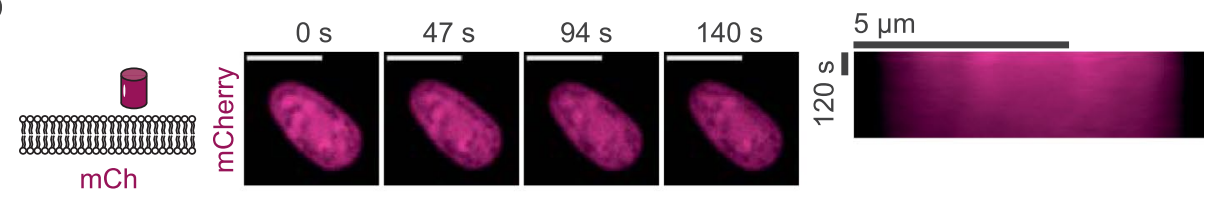

$0 \mathrm{~s}$

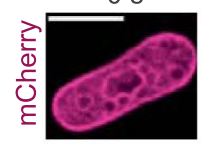

$46 \mathrm{~s}$

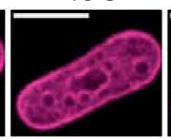

$92 \mathrm{~s}$

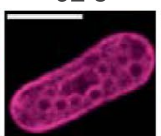

$139 \mathrm{~s}$

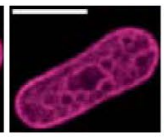

$184 \mathrm{~s}$

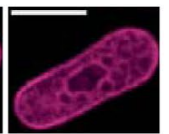

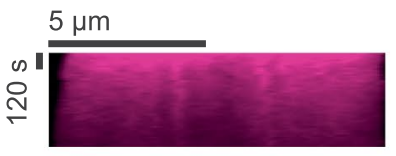

e

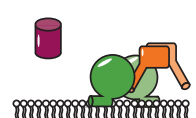

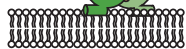

sfGFP-MinD/MinE $\mathrm{mCh}$

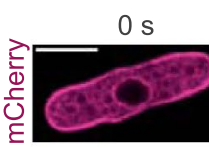

$47 \mathrm{~s}$

$93 \mathrm{~s}$

$138 \mathrm{~s}$
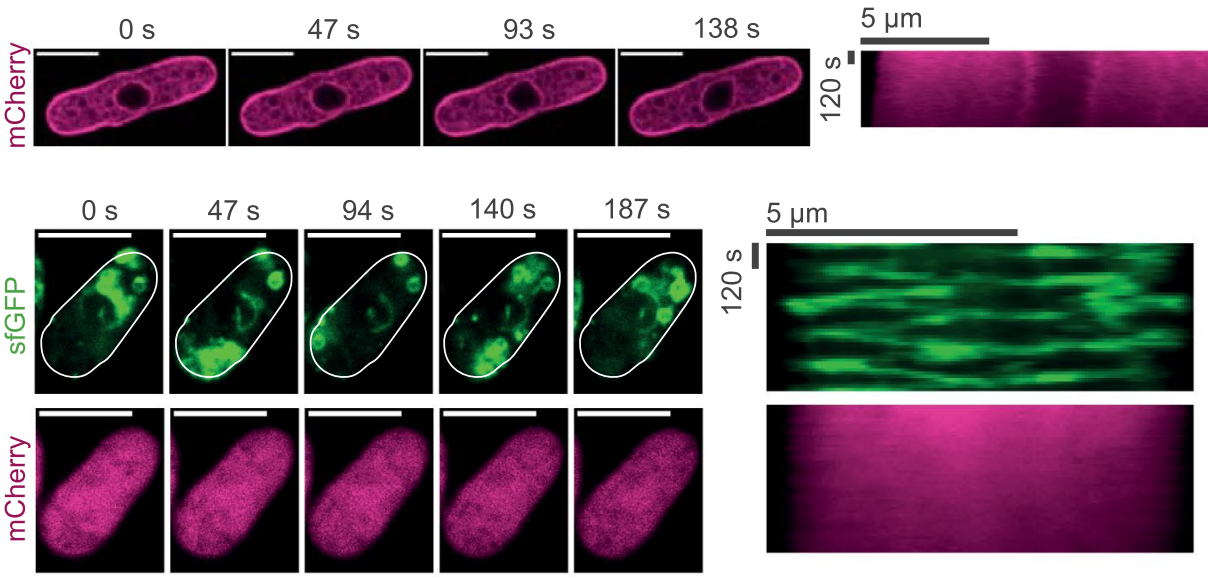

f
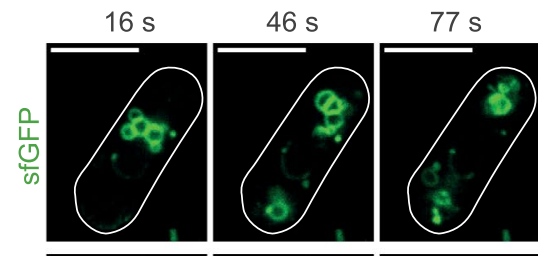

$107 \mathrm{~s}$
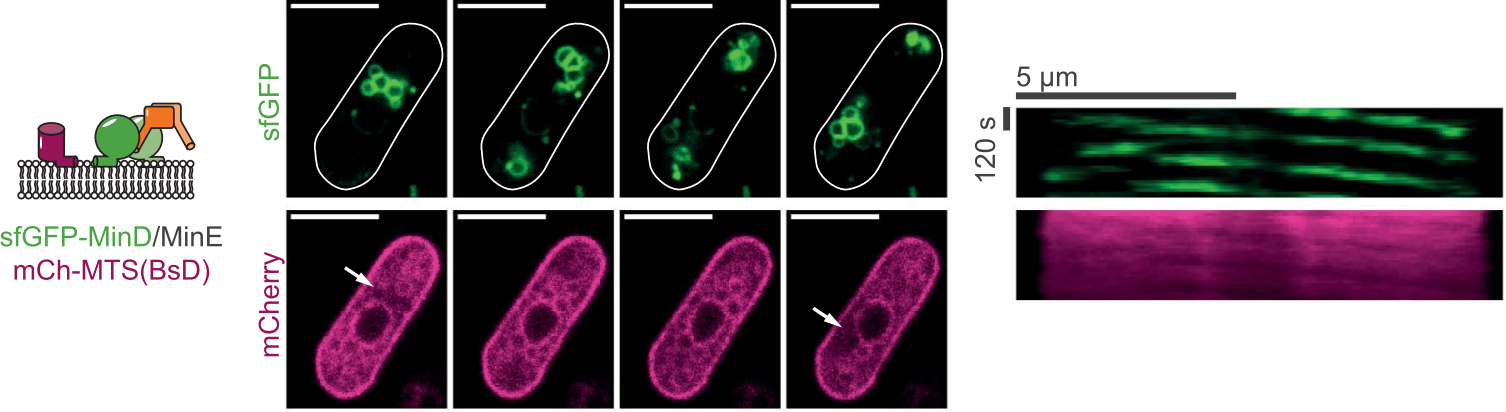

sfGFP-MinD/MinE mCh-MTS(BsD)

g
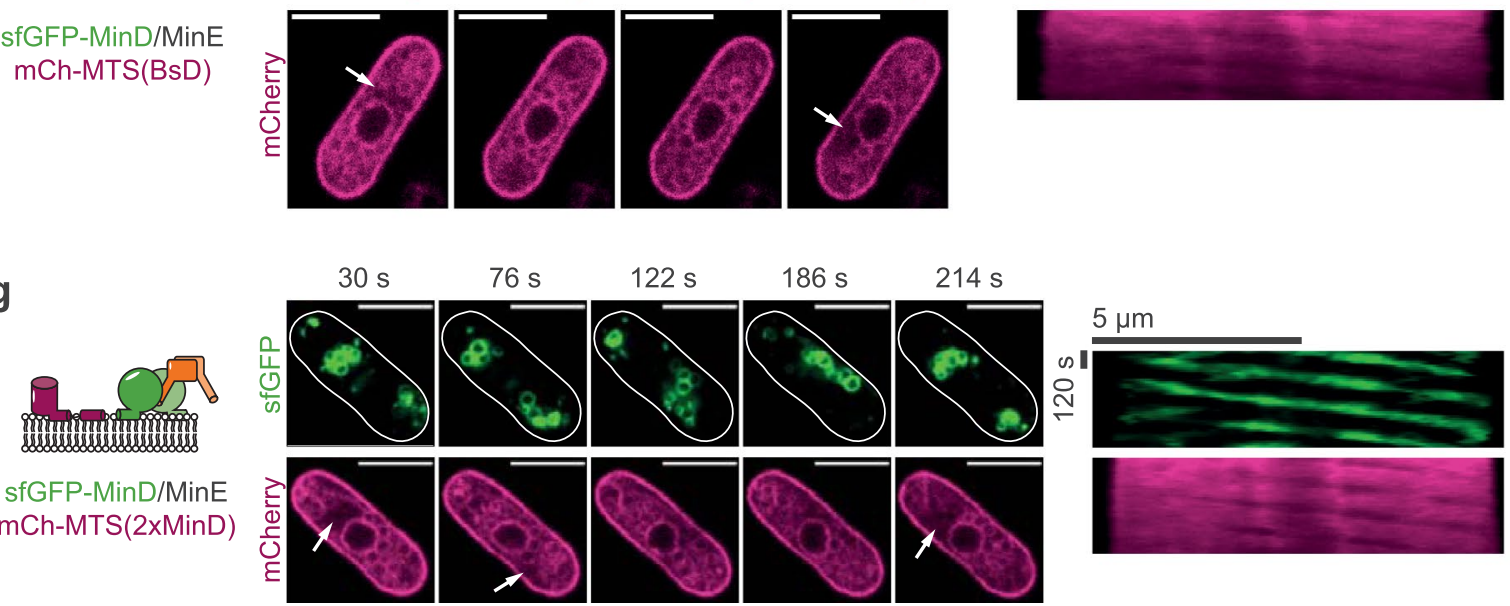

sfGFP-MinD/MinE mCh-MTS(2xMinD)

Extended Data Fig. 10 | MinDE spatiotemporally regulate model peripheral membrane proteins when reconstituted in S. pombe. a, Schematic of the plasmid used for expression of mCherry versions in S. pombe. Representative time-lapse and kymographs (over the entire cell length) of S. pombe cells expressing b, soluble mCherry, mCh which remains cytoplasmic, or a model peripheral membrane protein c, mCh-MTS(BsD) and d, mCh-MTS(2xMinD) which bind to membranes. Representative time-lapse and kymographs of S. pombe cells heterologously expressing sfGFP-MinD, MinE and $\mathbf{e}, \mathrm{mCh}$ or $\mathbf{f}$, mCh-MTS(BsD) or $\mathbf{g}$, mCh-MTS(2xMinD). MinDE self-organization spatiotemporally regulates the model peripheral membrane proteins mCh-MTS(BsD) and $\mathrm{mCh}-\mathrm{MTS}(2 \times \mathrm{Min} \mathrm{D})$ highlighted by white arrows, but not $\mathrm{mCh}$. Panel $\mathrm{g}$ is identical to Fig. $5 f$. White boundaries represent cell outline obtained from average fluorescence images. Scale bars, $5 \mu \mathrm{m}$. 


\section{Reporting Summary}

Nature Research wishes to improve the reproducibility of the work that we publish. This form provides structure for consistency and transparency in reporting. For further information on Nature Research policies, see our Editorial Policies and the Editorial Policy Checklist.

\section{Statistics}

For all statistical analyses, confirm that the following items are present in the figure legend, table legend, main text, or Methods section.

n/a Confirmed

$\triangle$ The exact sample size $(n)$ for each experimental group/condition, given as a discrete number and unit of measurement

$\square \bigotimes$ A statement on whether measurements were taken from distinct samples or whether the same sample was measured repeatedly

X The statistical test(s) used AND whether they are one- or two-sided

$\triangle$ Only common tests should be described solely by name; describe more complex techniques in the Methods section.

$\bigotimes \square$ A description of all covariates tested

Х $\square$ A description of any assumptions or corrections, such as tests of normality and adjustment for multiple comparisons

$\triangle$ A full description of the statistical parameters including central tendency (e.g. means) or other basic estimates (e.g. regression coefficient)

A $\square$ AND variation (e.g. standard deviation) or associated estimates of uncertainty (e.g. confidence intervals)

$\bigotimes \square \begin{aligned} & \text { For null hypothesis testing, the test statistic (e.g. } F, t, r \text { ) with confidence intervals, effect sizes, degrees of freedom and } P \text { value noted } \\ & \text { Give } P \text { values as exact values whenever suitable. }\end{aligned}$

Х $\square$ For Bayesian analysis, information on the choice of priors and Markov chain Monte Carlo settings

Х $\square$ For hierarchical and complex designs, identification of the appropriate level for tests and full reporting of outcomes

$\bigotimes \square$ Estimates of effect sizes (e.g. Cohen's d, Pearson's $r$ ), indicating how they were calculated

Our web collection on statistics for biologists contains articles on many of the points above.

\section{Software and code}

Policy information about availability of computer code

Data collection Zen Black (Zeiss), Andor Solis (Ver. 4.28, Andor 542 Technologies)

Data analysis Fiji (version v1.52p); Matlab (R2018a, The Math-Works, 546 Natick, USA); Origin 2018; GraphPadPrism8; Python (Python Software Foundation) with the following packages: numpy, scipy, pandas, sympy, Imfit, pims, matplotlib and bokeh

For manuscripts utilizing custom algorithms or software that are central to the research but not yet described in published literature, software must be made available to editors and

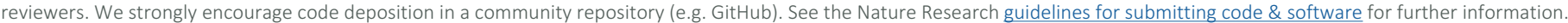

Data

Policy information about availability of data

All manuscripts must include a data availability statement. This statement should provide the following information, where applicable:

- Accession codes, unique identifiers, or web links for publicly available datasets

- A list of figures that have associated raw data

- A description of any restrictions on data availability

The raw images that support the findings of this study are available at Edmond with the identifier https://dx.doi.org/10.17617/3.5n or from the corresponding author upon request. 
Please select the one below that is the best fit for your research. If you are not sure, read the appropriate sections before making your selection.

\ Life sciences

Behavioural \& social sciences

$\square$ Ecological, evolutionary \& environmental sciences

For a reference copy of the document with all sections, see nature.com/documents/nr-reporting-summary-flat.pdf

\section{Life sciences study design}

All studies must disclose on these points even when the disclosure is negative.

Sample size $\begin{aligned} & \text { No sample size was calculated. Due to the large magnitude of the observed effects, we assume that our sample size is adequate. } \\ & \text { Data exclusions }\end{aligned}$
$\begin{aligned} & \text { Images were only excluded from analysis when supported lipid bilayers were defective or when the MinDE patterns were dynamic and not in } \\ & \text { the quasi-stationary state. For directed transport on chromium patterned membranes, membrane patches that did not show alignment of } \\ & \text { MinDE waves along the longest axis were excluded. }\end{aligned}$
Replication
Randomization numbers of experimental repeats are indicated in the respective figure legends. All experiments were performed at least in two or more
independent experiments with at least one sample chamber each under the same conditions, so that the total sample number was always $\geq 2$.

\section{Reporting for specific materials, systems and methods}

We require information from authors about some types of materials, experimental systems and methods used in many studies. Here, indicate whether each material, system or method listed is relevant to your study. If you are not sure if a list item applies to your research, read the appropriate section before selecting a response.

\begin{tabular}{|c|c|c|c|}
\hline \multicolumn{2}{|r|}{ Materials \& experimental systems } & \multicolumn{2}{|c|}{ Methods } \\
\hline$n / a$ & Involved in the study & $\mathrm{n} / \mathrm{a}$ & Involved in the study \\
\hline$\bigotimes$ & $\square$ Antibodies & Х & $\square$ ChIP-seq \\
\hline 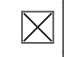 & $\square$ Eukaryotic cell lines & Х & $\square$ Flow cytometry \\
\hline$\bigotimes$ & $\square$ Palaeontology and archaeology & Х & $\square$ MRI-based neuroimaging \\
\hline$\bigotimes$ & $\square$ Animals and other organisms & & \\
\hline Х & Human research participants & & \\
\hline$\bigotimes$ & $\square$ Clinical data & & \\
\hline$\bigotimes$ & $\square$ Dual use research of concern & & \\
\hline
\end{tabular}

